\title{
Explanatory Item Response Models for Dyadic Data from Multiple Groups*
}

\author{
James P. Murphy ${ }^{\dagger}$ \\ Institute for Policy Research
}

Northwestern University

FORTHCOMING IN SOCIOLOGICAL METHODOLOGY

Keywords: social networks; dyadic data; item response theory; multilevel

(hierarchical/mixed effects) models

Word Count: 8,950

\footnotetext{
* I am especially grateful to John Levi Martin for his guidance and thoughtful advice throughout the development of this project. I also thank Peter McMahan and Steve Raudenbush for valuable comments on earlier drafts, Adam Slez for a helpful conversation, and Sociological Methodology's reviewers for their meticulous critiques, which have greatly improved the paper.

This research uses data from Add Health, a program project designed by J. Richard Udry, Peter S. Bearman, and Kathleen Mullan Harris, and funded by a grant P01-HD31921 from the Eunice Kennedy Shriver National Institute of Child Health and Human Development, with cooperative funding from 17 other agencies. Special acknowledgment is due Ronald R. Rindfuss and Barbara Entwisle for assistance in the original design. Persons interested in obtaining Data Files from Add Health should contact Add Health, The University of North Carolina at Chapel Hill, Carolina Population Center, 206 W. Franklin Street, Chapel Hill, NC 27516-2524 (addhealth contracts@unc.edu). No direct support was received from grant P01-HD31921 for this analysis.

$\dagger$ James Murphy, Institute for Policy Research, Northwestern University, 2040 Sheridan Road, Evanston, IL 60208. Email: james.murphy@northwestern.edu
} 


\begin{abstract}
$\underline{\text { Abstract }}$
Like other quantitative social scientists, network researchers benefit from pooling information from multiple observed variables to infer underlying (latent) attributes or social processes. Appropriate network data for this task is increasingly available. The inherent dependencies in relational data, however, pose unique challenges. This is especially true for those involved in the ascendant tasks of cross-network comparisons or multilevel network analysis. The author draws on item response theory and multilevel (mixed effects) modeling to propose a methodological approach that accounts for these dependencies and allows the analyst to model variation of latent dyadic traits across relations, actors, and groups precisely and parsimoniously. Examples demonstrate the approach's utility for three important research areas: tie strength in adolescent friendships, group differences in how discussing personal problems relates to tie strength, and the analysis of multiple relations.
\end{abstract}




\section{Introduction}

Social scientists frequently benefit from aggregating information on several directly observed variables to measure a common underlying (latent) trait or process, especially in survey analysis. This is no less true of social network researchers. If several observed variables are manifestations of a common latent trait, modeling them separately produces imprecise results. Some reduction in dimensionality is advisable. Network surveys frequently employ multiple questions eliciting different sorts of relationships and different relational qualities, which researchers then combine in some manner (e.g. Burt 1997; Marsden and Campbell 1984).

Item response theory (IRT) is the predominant approach to latent trait measurement in contemporary psychometrics. IRT conceives of people's responses to items on surveys, tests, and similar instruments as a function both of underlying traits of (usually but not necessarily ${ }^{1}$ ) individuals and properties of the items themselves. This vision of the response process contrasts with classical test theory (CTT) methods-like the ubiquitous summative scale - that assume responses are the product solely of an individual trait and random error that is not systematically related to the questions asked. Although more common in educational and psychological measurement, some sociologists have noted the advantages of IRT for survey research (Duncan 1985a, 1985b; Raudenbush, Johnson, and Sampson 2003; Raudenbush and Sampson 1999).

\footnotetext{
${ }^{1}$ For example, Raudenbush and Sampson's (1999) unit of analysis is the city block and the "items" are the presence or absence of a variety of physical features and social activities as recorded by trained observers.
} 
Separately, network researchers have become increasingly attune to the need for multilevel analyses of networks from multiple groups. This literature contributes new insights to the generalizability and variability of network processes (Snijders 2016). Comparable measurement across different sites should be of particular concern in such studies.

Past work has shown that item response models can be represented within the multilevel (hierarchical or mixed effects) modeling framework familiar to quantitative sociologists (de Boeck and Wilson 2004; Raudenbush and Sampson 1999). This representation allows for contextual variation and incorporation of explanatory variables to model latent traits. I extend this logic to dyadic data with dichotomous observed item responses.

Conceptually, the proposed methods treat dyads (pairs of actors) as cross-nested within two actors who - depending on the study design — themselves are potentially nested within a group. Within the dyad, a continuous latent trait generates observed responses to survey questions (or analogous instruments) in which one actor describes their relationship with the other. The proposed methods distinguish between contributions attributable to each actor, the specifics of their relationship, and (when applicable) group context. The multilevel representation allows analysts to parsimoniously test theoretical explanations of the underlying process while simultaneously accounting for measurement error. Moreover, because the IRT paradigm attends to properties of the specific items asked, an analyst can explore how different descriptions of the same dyad relate to each 
other and, broadly speaking, test explanations for why otherwise similar relationships are described in different ways.

I begin with an introduction to the basic framework in a single group setting. Section II develops two dyadic measurement models for network surveys and applies them to examine friendship strength among adolescents in a large high school in the National Longitudinal Study of Adolescent Health. Section III extends the framework to accommodate multiple groups. Section IV incorporates explanatory covariates to model how latent dyadic traits depend upon actor, dyad, and contextual factors. An example application shows how gender-gaps in friendship strength vary across schools with different organizational characteristics.

Sections V and VI extend the framework to more advanced applications. First, differential item functioning (DIF) facilitates an analysis of how the relational significance of discussing personal problems depends on adolescent dyads' gender composition. Section VI demonstrates how to model multiple latent relations simultaneously using data on personality attributions in 44 naturally occurring communities. Section VII concludes with a discussion of potential extensions.

\section{Measurement Models for Dichotomous Items}

IRT's conception of the response process differs fundamentally from the classical test theory (CTT) tradition still common in sociology. CTT assumes a generative model in which observed item responses are solely the product of an individual's level of a latent trait ("true score") and random error unrelated to the specific items in an 
instrument. IRT's generative model treats responses as the joint function of the trait and properties of the specific items asked. This simple observation has several advantageous implications for measurement including but not limited to: trait scores are generalizable beyond the specific instrument administered; item properties are generalizable beyond the specific sample of respondents; and reliability is not determined by the number of items administered (Duncan 1985b; Embretson 1996; Rusch et al. 2017).

Existing work on network measurement error operates within the CTT paradigm and focuses on reliability and validity in two settings. The first considers respondents' accuracy in discerning a latent "true" network (Butts 2003; Iacobucci, Neelamegham, and Hopkins 1999; Lee and Butts 2018).

More directly related to this paper's tasks, other scholars have examined the reliability and validity of composite dyadic measures from an actor's responses to multiple items. Ferligoj and Hlebec $(1995,1999)$ and Kogovšek et al. (2002) evaluated used repeated measurements experiments to analyze the reliability of social support items under different response scales data collection techniques (e.g. nomination from lists of alters versus free recall), and response modes (e.g. face-to-face versus telephone interviews). Recently, Koehly and Marcum (2018) introduced several CTT-inspired indices to assess the reliability and validity of dichotomous items as measures of latent relational qualities. These studies have not, however, accounted for the inherent dependencies in network data. IRT generalizes the core concepts of reliability and 
validity. ${ }^{2}$ Thus, this paper builds on and extends to different ends previous approaches employing multiple indicators of network ties.

In addition to introducing an IRT formulation to inform research on network survey response, this paper's main contributions lie in formulating explanatory (structural) models of both latent network traits and properties of survey items. I also demonstrate the benefits of this framework for research designs with multiple groups, increasingly common in network research (Snijders 2016). Prior work on multiple-item designs has not (1) accounted for the inherent dependencies in network data, (2) synthesized measurement and explanatory models, or (3) modeled how item properties vary across respondents (due to prior methods' reliance on CTT). Several approaches to respondent accuracy have integrated measurement models with structural models of the latent network, but — excepting Iacobucci et al. (1999) — they each rely on a single item to tap the latent network. Past network measurement models have not explicitly accommodated multiple group designs.

This section develops two dyadic IRT models that allows for the decomposition of a single latent trait into actor and dyadic components based on dichotomous items. I show how treating dyads as cross-classified data structures allows us to formulate a multilevel model in which a latent trait is captured by a series of random effects. I develop Rasch and two-parameter logistic models that explore different aspects of the

\footnotetext{
${ }^{2}$ Rusch et al. (2017) contrast the CTT and IRT conceptions of validity and reliability for social scientists.
} 
trait and the items used to measure it, applying both models to examine adolescent friendship strength in a large high school.

\section{$\underline{\text { Rasch Model }}$}

The canonical item response model is the Rasch or one-parameter logistic model. $Y_{m j}$ is a dichotomous variable for person $j$ 's response to an item $m$. For a positive response by person $j$ to item $m, Y_{m j}=1$. The log-odds of a positive response are represented by $\eta_{m j}$.

$$
\eta_{m j}=\pi_{j}-\psi_{m}
$$

Thus, $\eta_{m j}$ is a function of both respondent $j$ 's latent trait score $\left(\pi_{j}\right)$ and an item-specific "difficulty," $\left(\psi_{m}\right)$ characterizing the chance of responding positively to the item, conditional on the respondent-specific trait score. For a constant level of the latent trait, the odds of a positive response are lower for more difficult items.

The Rasch model entails two key assumptions. Local independence holds that, conditional upon $\pi_{j}$ and $\psi_{m}, Y_{m j}$ is drawn independently and identically from a Bernoulli distribution. The second major assumption is that differences between persons $\left(\pi_{j}\right)$ and differences between items $\left(\psi_{m}\right)$ contribute additively to the log-odds of an affirmative response. The implication is that items with less frequent positive responses are more difficult. 


\section{Rasch Models as Multilevel Models}

In the Rasch model's classic formulation, both person scores and item difficulties are fixed effects. It is possible to instead treat $\pi_{j}$ and $\psi_{m}$ as draws from random distributions. Thus, the Rasch model is a generalized linear mixed (multilevel) model. In this paper I treat only the person scores $\left(\pi_{j}\right)$ as random effects, leaving item parameters as fixed effects. ${ }^{3}$

I distinguish between items (questions asked) and responses (the act of answering a question). This distinction facilitates including respondents who answer some but not all items. Item-level missingness is difficult to avoid in practice and excluding respondents with partial data risks biasing results. An advantage of multilevel item response models is that by treating traits as random, we can pool information across respondents. Resulting estimates are unbiased when, conditional upon responses to observed items and explanatory covariates, item-missingness is unrelated to the unobserved response. This is a relatively weak assumption when observed data contain substantial information (Raudenbush et al. 2003).

In this paper's empirical examples, respondents answer all items. Nonetheless, my notation distinguishes between response and item to underscore the possibility of partial missingness. Items and responses are indexed by $m$ and $i$, respectively.

\footnotetext{
${ }^{3}$ See van den Noortgate and de Boeck's (2005) discussion of random item effects.
} 
The response process can now be represented as a two-level model with item responses (level-1) nested within individuals (level-2). The level-1 model is a measurement model. For consistency with more familiar generalized linear model notation, I replace "difficulty" of item $m$ with its "easiness" $\left(\delta_{m j}\right)$, the additive inverse of difficulty $\left(\delta_{m j}=-1 * \psi_{m j}\right)$.

$$
\eta_{i m j}=\pi_{j}+\sum_{m=1}^{M-1} \delta_{m j} D_{m i j}
$$

$D_{m i j}$ is a dummy variable equaling 1 if response $i$ is to item $m$. For $M$ items, the equation includes $M-1$ item dummies, leaving one item as a reference category. Person $j$ 's score on the latent trait is represented by $\pi_{j}$, expressed as the log-odds of $j$ responding positively to the omitted item. $\delta_{m j}$ is item $m$ 's easiness relative to the reference item: positive values of $\delta_{m j}$ indicate that for a constant level of trait $\pi_{j}$, people are more likely to provide a positive response to $m$ than to the reference item.

Moving to the person-level, $(2 \mathrm{~b})$ represents person $j$ 's level of the latent trait $\left(\pi_{j}\right)$ as the population average $\left(\beta_{0}\right)$ plus a person-specific random effect $\left(u_{j}\right)$. Equation $(2 \mathrm{c})$ expresses the assumption that each item's easiness $\left(\delta_{m}\right)$ is constant across persons. ${ }^{4}$

\footnotetext{
${ }^{4}$ For identification, one must either omit a reference item or constrain the intercept to zero. In the latter case, one includes dummy variables for all $M$ item, making the trait's unit of measurement the log-odds of a positive response to a hypothetical "average item" with $\delta_{m}$ directly interpretable as item easiness rather than relative easiness. Deciding between omitting a reference item or constraining the intercept is an aesthetic choice: both are identifiable and estimates under one representation can be derived from the other.
} 


$$
\begin{aligned}
& \pi_{j}=\beta_{0}+u_{j} \\
& \delta_{m j}=\delta_{m}, m=1 \ldots, M-1
\end{aligned}
$$

The typical possibilities of multilevel models are now open. As we shall see in Section IV, covariates may be incorporated at level-2 to predict variation in the latent trait across respondents, what de Boeck and Wilson (2004) term explanatory item response models. Moreover, additional nesting of units can be accommodated. For example, Raudenbush et al. (2003) formulate a three-level model with item responses nested within persons nested within city neighborhoods.

Network data does not, however, lend itself to such strict, hierarchical nesting. An alternative formulation is necessary.

\section{Dyads as Cross-classified Data Structures}

Network researchers are typically forced to characterize a dyad with a single manifest variable. However, the targets of our theoretical statements are latent qualities that could be tapped in multiple potential ways (Koehly and Marcum 2018; Newman 2018). Measuring latent traits is the domain of IRT models, but to fully utilize them, we must recast them in a form appropriate for the inherent dependencies of network data. Luckily, a strand of multilevel modeling explicitly deals with analogous data.

Cross-classified data structures consist of lower-level observations nested within two or more types of clusters that are not strictly nested within each other. A familiar example is students (level-1) cross-nested within schools and neighborhoods (level-2): a 
school may draw students from several neighborhoods while neighborhoods may send students to several schools.

In the present case, responses are nested within a directed dyad from ego to alter. The data are cross-classified because each ego may send ties towards several alters and alters may receive ties from several egos. Item responses are strictly nested: each response belongs exclusively to one directed dyad.

Table 1 depicts a data structure like the National Longitudinal Study of Adolescent Health used in forthcoming examples. Students (egos) name other students (alters) they consider friends. Ego subsequently answers four questions describing their friendship with each alter. Some students reciprocate each other's nominations (e.g. Students 1 and 2). Other nominations go unreciprocated (e.g. Student 2 nominates Student 4 but not vice versa). Importantly, students in the set of egos are not necessarily in the set of alters and vice versa. Student 6 does not list any friends but is named as a friend by Students 2 and 5. Student 5 lists two friends but receives no nominations themselves. Student 5 is counted in the school's set of egos but not the set of alters; Student 6 is counted in the set of alters but not the set of egos.

\section{[TABLE 1 ABOUT HERE]}

Others have noted that dyads are cross-classified units amenable to mixed effects modeling (e.g. Hoff 2005, 2009; Lazega and van Duijn 1997; van Duijn, Snijders, and Zijlstra 2004; Hoff et al. 2013; Warner, Kenny, and Stoto 1979). These include multiple group extensions of the social relations model for observed interval-scale dyadic 
outcomes (Snijders and Kenny 1999) and the $p_{2}$ model for ties' observed presence or absence (Zijlstra, van Duijn, and Snijders 2006). The present paper shows how we can use a similar logic for latent trait analysis by nesting a measurement model within a structural multilevel model, allowing us to examine aspects of the measurement process, link the trait to explanatory covariates, and pool data from multiple groups.

To accommodate the cross-classification, we augment the earlier model.

Level-1:

$$
\eta_{i m(j, k)}=\pi_{(j, k)}+\sum_{m=1}^{M-1} \delta_{m(j, k)} D_{m i(j, k)}
$$

Level-2:

$$
\begin{aligned}
& \pi_{(j, k)}=\theta_{0}+b_{j}+c_{k}+d_{(j, k)} \\
& \delta_{m(j, k)}=\delta_{m}, m=1 \ldots, M-1
\end{aligned}
$$

The latent trait $\pi_{(j, k)}$ is a property of a directed dyad $(j, k)$, the unique combination of ego $j$ and alter $k$. The data's cross-classified structure is reflected in (3b). $\theta_{0}$ is the average level of the trait across all dyads. Instead of a single random effect as in (2b), the cross-classified model includes three: $b_{j}$ is the ego-specific contribution of $j$ towards latent tie strength, $c_{k}$ the alter-specific contribution, and $d_{(j, k)}$ the contribution unique to the directed dyad $(j, k)$. Both $b_{j}$ and $c_{k}$ are latent propensities that $j$ and $k$ contribute to every relation they participate in as ego or alter, respectively; $d_{(j, k)}$ is unique to their directed relation. (3c) reflects the assumption that each item's easiness is constant across egos, alters, and dyads. Assume the random effects $b_{j}, c_{k}$, and $d_{(j, k)}$ are independently and identically drawn from normal distributions with means zero and variances $\tau_{E G O}$, $\tau_{A L T E R}$, and $\tau_{D Y A D}$. We assume that, conditional on the random effects, dyads are 
independent of each other. All subsequent models maintain these distributional assumptions.

\section{Example. Tie Strength in Adolescent Friendships}

Since Granovetter's (1973) seminal article, tie strength has been a core topic in network analysis. It has, however, proven slippery to measure. Even in its original formulation, tie strength had the flavor of a latent trait: "a (probably linear) combination of the amount of time, the emotional intensity (mutual confiding), and the reciprocal services which characterize the tie" (1361). Marsden and Campbell (1984) showed that we may generally distinguish between two correlated but distinct types of strength: time spent in the relationship and its emotional intensity. I focus here upon the former, hereafter referred to as interactional strength.

Data

The National Longitudinal Study of Adolescent Health (Add Health) is a nationally representative survey of American high schools and feeder schools. During the 1994-95 school year, an in-school survey was administered to students in attendance in 144 middle and high schools. Students were asked to name up to five male and five female friends inside or outside of school. For within-school nominations, respondents chose from a roster of registered students, allowing unique identification of egos and alters. As network data are particularly sensitive to missingness, I limit my candidate schools to 112 in which at least 70 percent of students on the school roster responded to the survey and 70 percent of alters named also responded. 
This initial example uses one large school of roughly 1,600 students. For each alter, the respondent (ego) reported whether they had engaged in five types of contact with alter within a given time frame. Previous studies have used these items to operationalize tie strength, but, to the best of my knowledge, only with summative scales that do not reflect potential differences in the activities' demands or significance (e.g. Kreager and Haynie 2011; Payne and Cornwell 2007). As Tables 2 shows, the items' frequency distributions differ considerably, motivating an IRT approach accounting for item differences.

\section{[TABLE 2 ABOUT HERE]}

I include four items: whether ego (i) spent time with alter the previous weekend or, in the last seven days, (ii) visited alter's house, (iii) met with them after school to hang out, or (iv) spoke on the telephone. Confirmatory factor analyses indicated that this fouritem model was consistent with a single latent trait..$^{5}$ A subsequent example in Section V reintroduces the discarded item, discussing personal problems with alter.

\section{Estimation}

Standard maximum likelihood algorithms for multilevel models are computationally infeasible for high-dimensional models like those proposed here. I turn to Bayesian methods for parameter estimation, specifically Hamiltonian Monte Carlo

\footnotetext{
${ }^{5}$ When using all 112 schools, the model's root mean squared error of approximation (RMSEA) is .043 with a 95-percent confidence interval from .041 to .045. MacCallum, Browne, and Sugawara (1996) recommend below .05 as indicative of good fit and below 0.10 as acceptable.
} 
(HMC) via the No-U-Turn sampler (Hoffman and Gelman 2014), a form of Markov chain Monte Carlo (MCMC). The models were fit using the rstan and brms libraries for R (Buerkner 2016; Stan Development Team 2018).

Bayesian models require specifying a prior distribution for all parameters. I use weakly informative priors with wide dispersion parameters so that parameters' posterior means are influenced predominantly by the observed data and minimally by the priors. All fixed effects receive normal priors with mean zero and standard deviation 10. For random effects, I place half-Cauchy priors on their standard deviation, setting the location parameters equal to zero and scaling parameters equal to 2.5. Recent studies have shown that the half-Cauchy is a sensible default for non-informative priors for variance components, especially when there is reason to believe some variances may be small (Polson and Scott 2012).

For each model, I ran four separate chains with diffuse initial values to assess convergence upon the target posterior distribution. Each chain included 2,000 warmup iterations; the subsequent 2,000 iterations were retained. Thus, the reported results are based on 8,000 samples from the posterior distribution. Appendix A discusses and presents diagnostics for MCMC models.

\section{Results}

Table 3 reports a trait decomposition model as in Equations (3a) to (3c). The random components' standard deviations reflect differences in strength across egos, alters, and dyads. The bulk of variation in reported tie strength (63 percent) is attributable 
to dyad-specific differences - representing the particularities of a given friendshipfollowed at a distance by features of the respondent (ego; 32 percent). Alter-specific features account for only 6 percent of variation in tie strength. ${ }^{6}$

\section{[TABLE 3 ABOUT HERE]}

Turning to the measurement model's easiness parameters, the large negative estimates for visiting alter's house and spending time with alter over the weekend indicate that these activities set a much higher standard for underlying tie strength than the reference item, hanging out after school. Talking on the phone occupies a middle ground. We now consider a modification to the measurement model that can provide more information on how each item relates to tie strength.

\section{Dyadic Two-parameter Logistic Models}

The Rasch model assumes that responses are an additive function of the latent trait and item properties. Items may, however, differ not only in easiness, but also in how indicative they are of the trait. An alternative to the Rasch model is the two-parameter logistic (2PL) model, which adds a discrimination parameter. Items that magnify differences between respondents' latent trait scores are more discriminating, more

\footnotetext{
${ }^{6}$ Dyad-level: $\frac{1.627^{2}}{\left(1.627^{2}+1.162^{2}+.482^{2}\right)}=0.626$; ego-level: $\frac{1.162^{2}}{\left(1.627^{2}+1.162^{2}+.482^{2}\right)}=0.319$; alter-level: $\frac{0.482^{2}}{\left(1.627^{2}+1.162^{2}+.482^{2}\right)}=0.055$
} 
sharply distinguishing levels of the latent trait. Less discriminating items account for less inter-respondent variability in the trait.

In general terms - without a multilevel structure - the 2PL can be written as follows for person $j$ answering item $m$.

$$
\eta_{m j}=\pi_{j}+\delta_{m}+\alpha_{m} \pi_{j}
$$

As before, $\pi_{j}$ represents person $j$ 's level of the trait and $\delta_{m}$ is item $m$ 's easiness. The difference between the 2PL and Rasch models lies with the slope parameter $\alpha_{m}$, item $m$ 's discrimination. ${ }^{7}$ The $2 \mathrm{PL}$ is a generalized nonlinear mixed model due to the interaction of the trait and discrimination parameters.

We can imagine at least two formulations of the 2PL for dyadic data. The first would include a single discrimination parameter $\left(\alpha_{m}\right)$ for each item $m$. In the friendship strength example, $\alpha_{m}$ signals how well item $m$ distinguishes stronger friendships from weaker ones. This approach would not, however, differentiate between ego, alter, and dyad components of strength.

A more informative version distinguishes between item $m$ 's ability to discriminate each component. One parameter $\left(\alpha_{m}^{E G O}\right)$ captures $m$ 's ability to distinguish between egospecific tendencies towards reporting stronger ties. A second $\left(\alpha_{m}^{A L T E R}\right)$ represents $m$ 's

\footnotetext{
${ }^{7}$ The $2 \mathrm{PL}$ reduces to the Rasch model when discrimination parameters are constrained to equal each other, $\alpha_{1}=\alpha_{2} \ldots=\alpha_{M}=1$.
} 
ability to distinguish alter-specific contributions. A third $\left(\alpha_{m}^{D Y A D}\right)$ indicates $m$ 's ability to distinguish between dyad-specific contributions.

Level-1: $\quad \eta_{i m(j, k)}=\pi_{(j, k)}+\sum_{m=1}^{M} \omega_{m(j, k)} D_{m i(j, k)}$

Level-2: $\quad \pi_{(j, k)}=b_{j}+c_{k}+d_{(j, k)}$

$$
\omega_{m(j, k)}=\delta_{m}+\alpha_{m}^{E G O} b_{j}+\alpha_{m}^{A L T E R} c_{k}+\alpha_{m}^{D Y A D} d_{(j, k)}, \quad m=1 \ldots, M
$$

Note important contrasts from the Rasch model formalized in (3a)-(3c). First, (5a) replaces $\delta_{m(j, k)}$ with $\omega_{m(j, k)}$ for reasons that will soon be apparent. Equation (5b) decomposes the latent trait into ego, alter, and dyad components, but, in contrast to (3b), contains no intercept $\left(\theta_{0}=0\right)$.

Equation $(5 \mathrm{c})$ reflects the key addition of the $2 \mathrm{PL}$. In addition to an easiness parameter $\left(\delta_{m}\right)$, there are now three new parameters per item $\left(\alpha_{m}^{E G O}, \alpha_{m}^{A L T E R}, \alpha_{m}^{D Y A D}\right)$, reflecting item $m$ 's discrimination of ego-specific contributions to the trait $\left(b_{j}\right)$, alterspecific contributions $\left(c_{k}\right)$, and dyad-specific contributions $\left(d_{(j, k)}\right)$. We return now to the example school to demonstrate the added insight that the 2 PL model can provide.

\section{Estimation.}

Two-parameter models are unidentifiable without constraints on either the trait variance or item parameters. The most common solution is to constrain the trait distribution to have a standard normal distribution, $\mathrm{N}(0,1)$. This would, however, prevent freely decomposing strength variance into ego, alter, and dyad components, an important motivation for adopting the multilevel IRT perspective for network data. Instead, I 
constrain one item's easiness parameters' to zero and one item's discrimination

parameters to 1 (Fox and Glas 2001:275).

Additionally, in these examples, all alter discrimination parameters $\left(\alpha_{m}^{A L T E R}\right)$ are constrained to one. Models freely estimating these parameters encountered severe difficulties in Markov chains' mixing, generating unreliable results. This is unsurprising given how little alter-level variance the initial Rasch model detected.

I to fit the model via HMC, using 4 chains of 6,000 iterations each, the first 1,000 for warmup. Following Fox (2010), the discrimination parameters receive log-normal priors with mean .5 and standard deviation 1 .

Results

Figure 1 presents the 2PL results via item characteristic curves (ICCs). (Appendix B includes full model results and diagnostics.) An ICC shows the probability of a positive response to item $m$ (y-axis) as a function of the underlying latent trait (xaxis). Discrimination is reflected in the shape of an ICC. More discriminating items have steeper gradients. Differences in item easiness are reflected in the curves' position at the average level of the latent trait ( 0 on the $\mathrm{x}$-axis). As mentioned above, with these data, computation necessitated constraining all alter discrimination parameters to equal one. Consequently, I only depict ego and dyad ICCs.

[FIGURE 1 ABOUT HERE] 
Items differ considerably in their ability to discriminate ego-specific tendencies towards strong and weak friendships. The ICCs in the top panel suggest that reporting spending weekend time with a friend is the act that best distinguishes respondents who tend to report stronger friendships from those that report weaker ones. Reporting visits to alter's house is also better at discriminating ego-specific contributions to tie strength than either hanging out after school or talking on the phone.

Differences between items' discriminations parameters for the dyad-specific component of strength (bottom panel) are less dramatic. Hanging out after school, talking on the phone, and spending time together on the weekend are equally adept at distinguishing dyad-specific strength specific. Hanging out after school is a slightly less discriminating.

The 2PL model offers important insights into the measurement properties of tie strength items. Activities that prima facie require more time or planning are both more difficult and more discriminating. However, items distinguish themselves from each other more in their ability to differentiate between respondents' varying propensities for reporting strong friendships in general than their ability to differentiate strength unique to a specific friendship.

The models developed in the remainder of the paper build from the dyadic Rasch model (Equations 3a-3c). The Rasch model's simpler linear form provides a clearer foundation for the extensions to follow than the 2PL. Moreover, the 2PL's nonlinear parameterization makes it considerably more intensive computationally. As shown in the 
online supplement, the models' Markov chains mix inefficiently. As we scale up to accommodate multiple groups and explanatory covariates, the much higher dimensionality and additional nonlinear terms would make parameter estimation impractical. ${ }^{8}$ However, in principle, the models that follow can accommodate item discrimination parameters.

\section{Extending to Multiple Groups}

Several considerations motivate extending the dyadic IRT model to multiple group designs. Empirical studies of contextual variation across networks are increasingly common (Snijders 2016). Such studies are theoretically important and assess the generalizability of network theories. Moreover, in multiple group designs we should be particularly concerned about the comparability of measurement properties across settings as they affect whether our instruments are truly gauging the same trait in the different groups.

Additionally, while the networks in Add Health are relatively large, encompassing entire schools, most network surveys do not enjoy this luxury. For example, studies of adolescents frequently gather data from multiple classrooms rather than schools (e.g. Smith et al. 2016). The relatively few actors in any single network (10s rather than 100 s) severely restricts the number of individual or dyadic covariates one might consider in

\footnotetext{
${ }^{8}$ Compared to educational and psychological tests, social surveys usually include far fewer items that tap a given latent trait. I suspect the computational difficulties encountered with the 2PL model for Add Health are likely to be the norm in applied settings.
} 
explanatory models - discussed in Section IV — thereby limiting the research questions that might be pursued. By pooling data from multiple groups, multilevel IRT models can overcome such challenges.

Approaching IRT from a multilevel perspective allows for nesting levels when data from multiple groups are available. This first allow for further trait decomposition into actor, dyad, and group components. Moreover, as fixed effects in multilevel models are shrinkage estimators, pooling groups in a single model generally provides more efficient parameter estimation than one would obtain applying the models separately by group (Fox 2010:151).

\section{Trait Decomposition}

Equations (3a)-(3c) showed how a latent trait like tie strength can be separated into ego, alter, and dyad components. With multiple groups we can also quantify variation attributable to group differences. Subscript $g$ indexes groups.

Level-1:

$$
\eta_{i m(j, k) g}=\pi_{(j, k) g}+\sum_{m=1}^{M-1} \delta_{m(j, k) g} D_{m i(j, k) g}
$$

Level-2:

$$
\pi_{(j, k) g}=\theta_{0 g}+b_{j g}+c_{k g}+d_{(j, k) g}
$$

Level-3:

$$
\begin{aligned}
& \theta_{0 g}=\gamma_{0}+h_{g} \\
& \delta_{m(j, k) g}=\delta_{m}, m=1 \ldots, M-1
\end{aligned}
$$

The measurement model in (6a) remains unchanged from (3a). At level-2, $\theta_{0 g}$ now represents the average level of the trait for dyads within school $g$. At level-3, $\gamma_{0}$ represents the average across all dyads, and the random effect $h_{g}$ is how much the 
strength of group $g$ 's average dyad deviates from $\gamma_{0}$. Assume $h_{g}$ is independently and identically drawn from a normal distribution with mean zero and variance $\tau_{\text {GROUP }}$.

As an example, we now consider cross-school variation in tie strength in Add Health. Recent studies have begun to examine how schools' organizational characteristics shape friendship formation (Frank, Muller, and Mueller 2013; McFarland et al. 2014). I quantify between-school variance in average friendship strength before proceeding in Section IV to models to account for cross-school variation.

\section{Sample and Estimation}

Due to computational constraints, these analyses rely on 50 schools randomly selected from the 112 that met the response rate criteria mentioned in Section II. These 50 schools include over 540,000 item responses describing almost 110,000 directed dyads.

MCMC methods are computationally impractical at this scale. I turn to integrated nested Laplace approximation (INLA), a recently developed alternative which performs Bayesian inference at much faster speeds than MCMC. ${ }^{9}$ In simulation studies, INLA has been shown to be at least as accurate as MCMC for most problems (Rue et al. 2017; Rue, Martino, and Chopin 2009; Wang, Yue, and Faraway 2018). The models were fit using the R-INLA library (Rue et al. 2017). As INLA relies on numerical integration to

\footnotetext{
${ }^{9}$ It bears mentioning that generalized nonlinear mixed models like the 2PL do not belong to the class of models that can be fit with INLA (latent Gaussian models). For nonlinear models, variational approximation methods can be faster alternatives to MCMC (Blei, Kucukelbir, and McAuliffe 2017).
} 
approximate the posterior rather than sampling the posterior like MCMC, diagnostics in Appendix A are inapplicable. ${ }^{10}$

\section{Results}

The trait decomposition model (Table 4) suggests similar levels of ego, alter, and dyad variance as in the example school. In addition, we see that schools account for as much variance in tie strength as alters themselves. Having distinguished four sources of strength, we now consider how to account for this variation and examine how the importance of actor and dyadic factors may vary across contexts.

\section{[TABLE 4 ABOUT HERE]}

\section{$\underline{\text { IV. Explanatory Models }}$}

Sociologists are usually interested in latent traits as either outcomes or predictors in explanatory model. In such cases - regardless of whether we have more than one group — multilevel IRT models provide additional advantages over CTT approaches. While a sum score is a sufficient statistic for a Rasch trait score, the two are not linearly related. Thus, when generalized linear models are applied to sum scores for explanatory purposes they may yield misleading results (Duncan 1985a:224).

\footnotetext{
${ }^{10}$ The closest analogue for INLA is the parameters' Kullback-Leibler (K-L) divergence. Smaller values indicate more accurate approximations to the target posterior. The K-L divergence is effectively zero for all parameters in all INLA models in this paper.
} 
Multilevel IRT models accommodate explanatory covariates at level-2 and above. By nesting a measurement model within the explanatory model, one avoids standard linear models' assumption of zero measurement error in the outcome, a problematic assumption, especially when dealing with latent traits. Moreover, because fixed effects in multilevel models are shrinkage estimators, they are generally more efficient than those in conventional models (Fox 2010:151).

I present the explanatory dyadic IRT model by example, focusing on how friendship strength varies with the gender mix of dyads. After establishing baseline differences, I examine how gender gaps vary across schools with different organizational features.

Adding Actor and Dyad Covariates.

We may represent tie strength's association with gender mix as follows.

Level-1:

$$
\eta_{i m(j, k) g}=\pi_{(j, k) g}+\sum_{m=1}^{M-1} \delta_{m(j, k) g} D_{m i(j, k) g}
$$

Level-2:

$$
\begin{aligned}
& \pi_{(j, k) g}=\theta_{0 g}+\theta_{1 g} \operatorname{GIRLBOY} Y_{(j, k)}+\theta_{2 g} B O Y G I R L_{(j, k)} \theta_{3 g} B O Y B O Y_{(j, k)} \\
& +b_{j g}+c_{k g}+d_{(j, k) g}
\end{aligned}
$$

Level-3:

$$
\theta_{0 g}=\gamma_{0}+h_{g}
$$




$$
\begin{aligned}
& \theta_{1 g} \ldots, \theta_{3 g}=\gamma_{1}, \ldots, \gamma_{3} \\
& \delta_{m(j, k) g}=\delta_{m}, m=1 \ldots, M-1
\end{aligned}
$$

The interpretation of the level-1 model (7a) is the same as (6a). At level-2, $\theta_{0}$ now represents the average level of the trait for same-gender female dyads. $\theta_{1}, \theta_{2}$, and $\theta_{3}$ are gaps in tie strength between same-gender female dyads and, respectively, female ego/male alter, male ego/female alter, and same-gender male dyads. As indicated by (7d), these effects are (for now) assumed constant across schools.

The results of this model appear in Table 4 as the "gender mix model." A subsequent model adds actor and dyad control variables to (7b). These include gradelevel homophily and reciprocated friendship nominations ${ }^{11}$ as well as the number of shared friends. Actor covariates include ego's in-degree and out-degree, alter's in-degree, and a dummy variable for ego's grade level. I also include the interaction of ego's gradelevel with gender mix to adjust for age differences in friendship perception that may be related to gender (Rose and Rudolph 2006). All continuous variables were entered in the model as z-scores. The online supplement includes descriptive statistics.

\footnotetext{
${ }^{11}$ Note that the reciprocal gesture is naming the other student as a friend and does not require both students to name the same activities.
} 


\section{Results of Actor/Dyad Covariate Models}

Figure 2 displays fitted values for tie strength by gender mix both with and without actor and dyad controls. The trait is scaled in terms of the probability ego reported hanging out with alter in the last week conditional on latent tie strength.

The strongest friendships are between girls, substantially stronger than friendships between boys. Mixed-gender relationships are similarly weak from both genders' perspectives. The pattern is little changed by including additional covariates.

\section{[FIGURE 2 ABOUT HERE]}

Perhaps surprisingly, although grade-level homophily is a well-established pattern for the existence of friendships (or, more precisely, naming friends), in the model including control variables it is negatively related to tie strength (Table 4). Speculatively, the potential barriers to cross-grade friendship may be sufficiently high that only interactionally strong friendships can be maintained across grade-levels. I will return to this observation in the conclusion.

\section{Modeling Tie Strength across Groups}

I turn now to whether some organizational factors related to tie formation also explain variation in tie strength. I examine three school-level features McFarland et al. (2014) found predicted between-school variation in friendship formation in Add Health.

Past work suggests school size will correspond with both stronger ties overall and larger gaps between the strength of same-gender friendships and mixed-gender 
friendships. Larger settings provide greater opportunities for homophily on diffuse characteristics like gender, reflected in the slopes for gender mix (McFarland et al. 2014).

Gender heterogeneity determines the availability of same-gender and crossgender friendships within the school. I measure heterogeneity using the inverse of the Herfindahl index. ${ }^{12}$ Finally, because of their differing age composition and organizational features, I distinguish between middle and high schools (Frank et al. 2013; McFarland et al. 2014). Add Health defines a high school as any school that includes an $11^{\text {th }}$ grade.

As an intermediate step, the "intercepts-as-outcomes" model includes characteristics as predictors of schools' average tie strength conditional on the actor and dyad covariates.

The ultimate model of interest is a "slopes-as-outcomes" model where school variables predict school-specific gender-gaps. ${ }^{13}$ Because high school students are on

${ }^{12}$ GENDERHETEROGENEITY $_{g}=1-\left(\right.$ PROPORTIONMALE $2+$ PROPORTIONFEMALE $\left.{ }_{g}^{2}\right)$

${ }^{13}$ A reviewer raised the important possibility that Add Health's cap on friendship nominations (5 per gender) might introduce a floor effect on strength. By removing low-strength friendships from the sample, the cap may produce misleading estimates. One way to explore this possibility is to re-run the slopes-asoutcomes model on the subset of students who name fewer than 5 same-gender friends (including both inschool and out-of-school friends), roughly 28 percent of respondents (egos) sampled. For this subsample, we need not fear that there are unobserved friendships that did not make the cut. These results appear in Appendix F. Support for many of the school-level effects discussed here dissipates when using this subsample, which may limit the generalizability of the findings reported in the main text. I also include a broader subset of students who name 4 or fewer in-school same-gender friends, which matches more 
average older than middle school students, I add the interaction of gender mix and ego's grade-level to avoid conflating organizational and age effects on the gender mix slopes. For brevity, I use vector notation (in bold) for the actor/dyad controls. Size and gender heterogeneity are transformed to z-scores.

Level-1:

$$
\eta_{i m(j, k) g}=\pi_{(j, k) g}+\sum_{m=1}^{M-1} \delta_{m(j, k) g} D_{m i(j, k) g}
$$

Level-2:

$$
\begin{aligned}
\pi_{(j, k) g} & =\theta_{0 g}+\theta_{1 g} \operatorname{GIRLBOY}_{(j, k)}+\theta_{2 g} \operatorname{BOYGIRL}_{(j, k)} \\
& +\theta_{3 g} \text { BOYBOY }_{(j, k)}+\boldsymbol{\theta}_{\mathbf{4 g}} \text { CONTROLS }_{(j, k)}+b_{j g}+c_{k g}+d_{(j, k) g}
\end{aligned}
$$

Level-3:

$$
\begin{aligned}
\theta_{0 g}= & \gamma_{00}+\gamma_{01} Z_{\text {ZENDERHETEROGENITY }}+\gamma_{02} \text { ZSIZE }_{g} \\
& +\gamma_{03} \text { MIDDLE SCHOOL }{ }_{g}+h_{0 g} \\
\theta_{1 g}= & \gamma_{10}+\gamma_{11} \text { ZGENDERHETEROGENITY }_{g}+\gamma_{12} \text { ZSIZE }_{g} \\
& +\gamma_{13} \text { MIDDLE SCHOOL }
\end{aligned}
$$

closely to the main analyses. The results here are intended as illustrations of dyadic IRT methods rather than exhaustive explorations of factors affecting tie strength. 


$$
\begin{aligned}
\theta_{2 g}= & \gamma_{20}+\gamma_{21} \text { ZGENDERHETEROGENITY } Y_{g}+\gamma_{22} \text { ZSIZE }_{g} \\
& +\gamma_{24} \text { MIDDLE SCHOOL } \\
\theta_{3 g}= & \gamma_{30}+\gamma_{31} \text { ZGENDERHETEROGENITY }_{g}+\gamma_{32} \text { ZSIZE }_{g} \\
& +\gamma_{34} \text { MIDDLE SCHOOL }_{g} \\
\boldsymbol{\theta}_{4 g} & =\gamma_{\mathbf{4}} \\
\delta_{m(j, k) g} & =\delta_{m}, m=1 \ldots, M-1
\end{aligned}
$$

\section{Results}

The online supplement includes the full parameter estimates for both the intercepts-as-outcomes and slopes-as-outcomes models. Figures 3, 4, and 5 show fitted values based on the slopes-as-outcomes model. The y-axis again expresses the latent trait in terms of the conditional probability of ego saying they hung out with alter outside of school in the last week.

As expected, friendships tend to be slightly stronger in larger schools. However, gender-based differences do not seem to depend on school size, reflected by the parallel lines in Figure 3.

\section{[FIGURE 3 ABOUT HERE]}

In contrast, gender composition appears related to gender gaps. Respondents generally report stronger relationships with their female friends, but the disparity is smaller in more heterogeneous schools. This is reflected in Figure 4, most notably in the 
steep slope for same-gender male dyads. It can also be seen in the gradual convergence of the lines for both types of mixed gender friendships. In short, perceptions of friendships with boys are stronger in more diverse settings.

\section{[FIGURE 4 ABOUT HERE]}

Schools' grade composition also appears implicated in gender gaps (Figure 5). Friendships in middle schools are generally stronger than those in high schools, but the gaps are more pronounced for cross-gender friendships. Girls' friendships with each other appear equally strong in middle and high schools.

\section{[FIGURE 5 ABOUT HERE]}

\section{$\underline{V}$. Differential Item Functioning}

Network survey items must, by necessity, label the sentiment, role, or behavior they intend to measure. Considerable debate exists about actors' interpretations of these labels' content and whether there is systematic variation in their interpretations (Burt 1997; Fischer 1982)

An important example involves the General Social Survey's “important matters" name generator, which asks respondents to name up to five alters with whom they discussed problems they considered important. Researchers initially assumed that this "core discussion network" represented strong ties, drawing conclusions about network size and segregation in America both cross-sectionally and longitudinally (e.g. McPherson, Smith-Lovin, and Brashears 2006). 
However, recent replications suggest that what constitutes an "important matter" is frequently of debatable importance (Bearman and Parigi 2004) and the people one discusses important matters are not necessarily personally important (Small 2013). In short, discussing important matters means different things to different people.

Differing interpretations of survey items exemplifies differential item functioning (DIF), the phenomenon of an item's easiness systematically varying across groups of people conditional upon having the same level of the underlying latent trait. This section approaches DIF as both a measurement issue and a substantive one. It modifies the previous dyadic IRT model to incorporate DIF to examine how the features of actors, dyads, and contexts may affect how much a given item aligns with a given dyadic trait like tie strength. I apply the dyadic DIF model to explore discussing personal matters in adolescent friendships.

DIF Models as Multilevel Models.

In standardized testing, DIF analyses are predominantly concerned with whether a given test item is biased against some groups relative to others. Differential impact exists if an item's easiness differs across groups when their members have equal levels of the latent trait (Meulders and Xie 2004).

In a multilevel framework, DIF is represented by treating both the trait and one or more level-1 slopes for item easiness as functions of higher-level covariates (van den Noortgate and de Boeck 2005). The following model assesses whether the easiness of Add Health's discussing personal problems item varies with dyadic gender mix. 
Level-1:

$$
\eta_{i m(j, k) g}=\pi_{(j, k) g}+\phi_{(j, k) g} P R O B L E M S_{i(j, k) g}+\sum_{m=1}^{M-1} \delta_{m(j, k) g} D_{m i(j, k) g}
$$

Level-2:

$$
\begin{aligned}
\pi_{(j, k) g} & =\theta_{0 g}+\theta_{1 g} \operatorname{GIRLBOY}_{(j, k)}+\theta_{2 g} \operatorname{BOYGIRL}_{(j, k)}+\theta_{3 g} \operatorname{BOYBOY}_{(j, k)} \\
& +b_{j g}+c_{k g}+d_{(j, k) g} \\
\phi_{(j, k) g} & =\lambda_{0 g}+\lambda_{1 g} \operatorname{GIRLBOY}_{(j, k)}+\lambda_{2 g} B O Y G I R L_{(j, k)}+\lambda_{3 g} B O Y B O Y_{(j, k)}
\end{aligned}
$$

Level-3:

$$
\begin{aligned}
& \theta_{0 g}=\gamma_{0}+h_{g} \\
& \theta_{1 g} \ldots, \theta_{3 g}=\gamma_{1}, \ldots, \gamma_{3} \\
& \delta_{m(j, k) g}=\delta_{m}, m=1 \ldots, M-1 \\
& \lambda_{0 g} \ldots, \lambda_{3 g}=\kappa_{00}, \ldots, \kappa_{30}
\end{aligned}
$$

At level-1, responses to the original items (anchor items) are indicated with $D_{m i(j, k) g}$ and their easiness represented by $\delta_{m(j, k) g}$. The trait (expressed as the conditional log-odds of hanging out with alter) remains $\pi_{(j, k) g}$. A new dummy variable $P R O B L E M S_{i(j, k) g}$ equals 1 if response $i$ is to the discussing personal problems item. The discussing personal problems item's differential impact is $\phi_{(j, k) g}$. In $(9 \mathrm{c})$, conditional on underlying strength, $\lambda_{0 \mathrm{~g}}$ is the relative easiness of the discussing problems item for same-gender female dyads in group $g$. Differences in group $g$ between the relative 
easiness for same-gender female dyads and other gender mixes are represented by $\lambda_{1 g}$,

$\lambda_{2 g}$, and $\lambda_{3 g}$. In (9g), the identity between each $\lambda_{\cdot g}$ and its corresponding $\kappa_{\cdot 0}$ reflects a temporary assumption that gender-based differential impact is constant across schools.

\section{DIF in Non-testing Contexts}

The idea of item bias in standardized tests is familiar. How should we think about differential impact of items in other response settings? Differential impact biases measurement, but we need not think of DIF as merely diagnostic. It teaches us about how a given latent trait aligns with other attitudes, activities, etc. among different sorts of people, allowing us to examine why an items' properties might be sensitive to qualities other than the latent trait. For example, if the item easiness for discussing personal problems with a friend varies with gender, then we have reason to believe that the social meaning of these discussions is gender-dependent. The question is not whether boys' or girls' understandings of friendship are "wrong" or misaligned. The question is whether their alignments differ and, consequently, under what conditions trait measurement will be comparable.

Consider Figure 6, which shows three hypothetical ICCs. The top and bottom curves show the relationship between tie strength and discussing personal problems for two different groups. The middle, dotted line represents a reference item ("hanging out" in this example), the easiness of which is the same for both groups. If no differential impact existed, the "discuss problems" lines for both groups would overlap. However, here we see that conditional on underlying strength, the item is easier for Group 1 than 
Group 2. This means Group 1's members have, on average, a lower bar for friendship strength compatible with discussing personal problems.

\section{[FIGURE 6 ABOUT HERE]}

Although DIF and multidimensionality are distinct issues generally, this situation constitutes what Douglas, Roussos, and Stout (1996) term "benign" DIF. The source of differential impact does not indicate a fault so much as it indicates that the item manifests both the focal trait (e.g. interactional tie strength) and an auxiliary trait, which the instrument lacks additional items to tap such as, in the present case, emotional tie strength. Differential impact is due to this auxiliary trait.

\section{Example}

Prior research suggests that self-disclosure is a more common path to intimacy among girls than boys (McNelles and Connolly 1999). A DIF model allows us to see whether this tendency persists after conditioning on interactional tie strength. If so, we can conclude that discussing problems' role in fostering close relationships depends upon the gender mix of the dyad. Table 5 reports parameter estimates from a baseline DIF model that includes dyadic gender mix as in (9a) to $(9 g)$ but excludes other explanatory covariates. $^{14}$

\section{[TABLE 5 ABOUT HERE]}

\footnotetext{
${ }^{14}$ The models for multiple groups use the same diffuse priors for fixed and random effects as the single school models.
} 
The baseline results illustrated in Figure 7 demonstrate that alignment of discussing problems and interactional strength is related to gender. The y-axis displays the easiness of the "problems" item for a given gender mix relative to the reference item ("hanging out"). Conceptually, in terms of Figure 6, this distance refers to the gap between a group's ICC for the discuss problems item and the ICC for the reference item at the average level of the trait ( 0 on the $\mathrm{x}$-axis). Positive values in Figure 7 indicate that, conditional on strength, the discussing problems item is easier than the reference item (hanging out) for that gender mix while negative values indicate it is more difficult.

Conditional on tie strength, girls are more likely to report discussing personal problems. Surprisingly, this tendency is roughly the same with both their male and female friends. In contrast, friends' gender is crucial for boys: in otherwise equally intensive friendships, boys are far less likely to discuss personal problems with other boys than with their female friends.

\section{[FIGURE 7 ABOUT HERE]}

We may add school covariates to $(9 \mathrm{~d}),(9 \mathrm{e})$, and $(9 \mathrm{~g})$ to explore cross-school variation. School size, gender heterogeneity, and type (high school or middle school) are

considered in the predictive model, adjusting for ego's grade level and its interaction with 
gender mix. ${ }^{15}$ I illustrate this model's key results visually; full parameter estimates appear in the online supplement.

Conditional on strength, discussing personal problems appears to be slightly more common in larger schools (Figure 8). The modest trend does not appear to depend upon the gender of ego or alter.

[FIGURE 8 ABOUT HERE]

However, gender heterogeneity appears to strongly affect the item's differential impact and amplifies gender differences. Figure 9 shows that, conditional on strength, discussing personal problems is rarer in more gender diverse schools. The decline is especially steep for friendships between boys.

\section{[FIGURE 9 ABOUT HERE]}

Figure 10 provides evidence that discussing personal problems' relation to strength differs between high schools and middle schools. Conditional on strength, discussing personal problems is less common in high school than middle school. The disparity does not appear to depend on friends' genders.

\section{[FIGURE 10 ABOUT HERE]}

\footnotetext{
${ }^{15}$ As with the results in Section IV, school-level effects are noisier when the sample is limited to students who name fewer than 5 friends. (See Appendix F.)
} 


\section{Modeling Multiple Traits}

Multiplexity — the propensity for pairs of actors to form multiple sorts of relationships with each other-is a longstanding sociological interest (Verbrugge 1979). We can extend the framework developed so far to multiple relations considered as two or more latent traits, opening new research opportunities. In addition to facilitating decomposition of traits into actor, dyad, and group components and constructing explanatory models, we can now examine the degree of multiplexity for each type of cluster-ego, alter, dyad, and group.

This section formalizes the decomposition of multiplexity at different levels and illustrates the procedure with data on personality attributions in 44 residential groups. Due to space constraints, I reserve for the online supplement an illustration of explanatory models for multiple traits, a straightforward extensions of the methods developed in Section IV.

\section{Multilevel IRT Models for Multiple Traits}

Raudenbush et al. (2003) proposed a multilevel Rasch model for two or more latent traits. This allows researchers to not only model the traits under consideration but also examine the traits' covariance. The correlation of the random effects at each level captures how tightly linked two traits are.

The key difference from earlier models is a reformulation of level-1. Building from the earlier multiple-group variant of the trait decomposition model (Equations 6a6d), $p$ indexes latent traits. 
Level-1:

$$
\eta_{i m p(j, k) g}=\sum_{p=1}^{P}\left(\pi_{p(j, k) g} A_{i m p}+\sum_{m=1}^{M-P} \delta_{m p(j, k) g} D_{i m p(j, k) g}\right)
$$

The level-1 intercept from earlier models has been removed. In its place, there are $P$ dummy variables $\left(A_{i m p}\right)$, one for each trait. If item $m$ taps trait $p, A_{\text {imp }}=1$. The parameter $\pi_{p(j, k) g}$ represents dyad $(j, k)$ 's level of trait $p$. Metaphorically, each $\pi_{p(j, k) g}$ is a distinct intercept that is "activated" if item $m$ manifests $p$. Note that there are $M-P$ item dummies as one reference item is omitted per trait.

For trait decomposition, the formulation at higher levels is essentially unchanged from the unidimensional model.

Level-2:

$$
\pi_{p(j, k) g}=\theta_{p 0 g}+b_{p j g}+c_{p k g}+d_{p(j, k) g}
$$

Level-3:

$$
\begin{aligned}
& \theta_{p 0 g}=\gamma_{p 0}+h_{p g} \\
& \delta_{p m(j, k) g}=\delta_{p m}, m=1 \ldots, M-p
\end{aligned}
$$

The degree of relations' multiplexity is given by correlations of random effects. For two latent traits, $\operatorname{cor}\left(b_{1 j g}, b_{2 j g}\right)$ gives the extent to which multiplexity is attributable to ego, $\operatorname{cor}\left(c_{1 \mathrm{~kg}}, c_{1 \mathrm{~kg}}\right)$ multiplexity due to alters, $\operatorname{cor}\left(d_{1(j, k) g}, d_{1(j, k) g}\right)$ multiplexity unique to dyads, and $\operatorname{cor}\left(h_{1 g}, h_{2 g}\right)$ multiplexity specific to groups. We assume $P$-by- $P$ multivariate normal covariance matrices for ego, alter, dyad, and group random effects, capturing both the variance of each trait and their correlation within each type of cluster. Example. Interpersonal Perception in Groups 
It is a classic sociological observation that groups shape their members' perceptions of each other (e.g. Mead 1967). Gender and race scholars have devoted much attention to attribution in the form of stereotyping. Yet interpersonal perception more generally and especially the role of group context in natural settings have received less empirical scrutiny.

Here I consider how the attribution of two personality traits, instrumentality and expressiveness. Instrumentality refers to a disposition towards autonomous control of one's environment; expressiveness entails selflessness and caring for others (Eagly and Steffen 1984). If ego (attributor) thinks alter (attributee) possesses a personality feature. there is a directed tie from ego to alter. As there are two latent relational traits to be measured, there exists a multiplex network of attributions.

\section{Urban Communes Data Set}

I leverage a unique data set to assess the preceding claims using the twodimensional multilevel dyadic IRT model. Zablocki's (1980) Urban Communes Data Set (UCDS) contains individual, relational, and group characteristics from 60 intentional communities in the United States in 1974. In the relational survey, each resident in the sampled communities was asked to name which group members they believed possessed several personality features. I use seven of these items: four to measure instrumentality and three for expressiveness. ${ }^{16}$ (See Table 6.) I limit analysis to attributees who were

\footnotetext{
${ }^{16}$ In confirmatory factor analyses, this 2-factor solution fit the data well. The RMSEA was 0.043 with a 95percent confidence interval from 0.035 to 0.052 .
} 
considered on relational surveys by at least 5 of their fellow members and groups with at least 5 unique attributees and 5 unique attributors. This restriction leaves 412 attributees evaluated by 350 attributors for a total of 3,277 attributor-attributee dyads in 44 groups.

\section{[TABLE 6 ABOUT HERE]}

\section{Model Estimation}

I return to HMC estimation for these models, using rstan and the rethinking libraries for R (McElreath 2016). Fixed effects received diffuse normal priors as in the Add Health models. For the random components, I use separate priors for the two traits' standard deviations (half-Cauchy priors) and their correlation. The correlation matrices' Cholesky factors take diffuse LKJ priors (Lewandowski, Kurowicka, and Joe 2009) with a shape parameter equal to $2 .{ }^{17}$ Four chains of 7,000 iterations each were run, devoting the first half of each chain to warmup.

Note that the group-level includes a random effect for expressiveness only. Earlier models showed so little group-level variance that including group random effects for both traits posed estimation problems. Hence, covariance of instrumentality and expressiveness are available only at the ego, alter, and dyad levels.

\section{Trait Decomposition and Covariance}

\footnotetext{
${ }^{17}$ A shape parameter of this magnitude discourages estimates of perfect correlation between random effects, both implausible and a barrier to efficient mixing of chains.
} 
We start with the variance decomposition model formulated in (10a) to (10d). Table 7 includes full results from this model. Note first that for instrumentality, the items for strong, decisive, or influential have essentially the same easiness. In contrast, being called dominant sets a much higher threshold for instrumentality. The easiness parameters are more differentiated for expressiveness: "supportive" has the lowest threshold (easiest) and "intuitive" the highest threshold (most difficult).

Our primary interests are the traits' variances and covariance at each level. Inspecting the random components (bottom of Table 8), attributions of instrumentality are predominantly due to attributee-specific effects. However, for expressiveness, attributee and dyad-specific variance are roughly equal. Attributees account for 69 percent of within-group variance of instrumentality but only 45 percent of expressiveness. ${ }^{18}$

While the trait variances suggest that attribution inheres largely in the attributee, the traits' correlation — the tendency towards multiplexity — is far stronger at the attributor level $(r=.84)$ than at the attributee or dyad level (.47 and .41 , respectively). In other words, associations between perceived instrumentality and expressiveness have less to do with aspects of attributees or relationships than with schemas individual attributors hold.

\section{[TABLE 8 ABOUT HERE]}

\footnotetext{
${ }^{18}$ For instrumentality $\frac{1.676^{2}}{.783^{2}+1.676^{2}+.810^{2}}=.689$; for expressiveness $\frac{1.260^{2}}{1.053^{2}+1.260^{2}+0.929^{2}}=0.446$
} 


\section{Discussion}

This paper introduced a multilevel IRT approach to latent trait measurement and modeling with dyadic data. These methods rest on a more realistic conception of survey response processes, feature well-established advantages for statistical and measurement properties, and open new opportunities for research on item properties through the likes of 2PL or DIF models, unexaminable under the CTT paradigm still prevalent in sociology. The multilevel representation naturally lends itself to testing ecological theories of networks (McFarland et al. 2014; Snijders 2016).

Several examples demonstrated the approach's utility. First, with Add Health, we observed that item discrimination differently affects measurement of ego-specific and dyad-specific contributions to reported friendship strength. Multiple group and explanatory models showed schools' organizational characteristics may differently affect same-gender and mixed-gender friendships as well as the salience of discussing personal problems in equally strong relationships. Finally, an example using multiple latent dyadic traits in 44 naturally occurring communities distinguished between multiplexity (correlation of traits) attributable to egos, alters, and dyads.

I have focused on analysis of dichotomous items, which are common in network surveys. Models for polytomous responses are possible. The standard models for ordered responses - partial credit and graded response models - are nonlinear like the 2PL and, given the cross-classified structure of dyadic data, may prove computationally impractical in many contexts. An alternative is the less common sequential ordered item 
response model (Jeon and de Boeck 2016), which, like the Rasch model, is linear in its parameters.

Using random effects to express higher-order dependencies is an additional area for development. Like the $p_{2}$ and the social relations models, the models developed above are (conditional) dyadic independence models. They assume - conditional on group, ego, alter, and dyad random effects_-dyads are independent of each other. Higher-order dependencies (e.g. triadic closure or clustering) must be expressed explicitly through covariates. An advantage of the multilevel approach is that it is mathematically straightforward to incorporate multiplicative random effects that capture higher-order dependencies (Hoff 2005, 2009). Such models will require customized algorithms for inference, a task beyond the scope of this paper.

Opportunities exist to jointly examine tie formation and intensity, for example, through item response tree approaches (Jeon and de Boeck 2016). For example, Section IV's Add Health analyses revealed strength was lower between friends in the same grade, a contrast with the homophily typically seen in friendship reports. Simultaneously modeling the friendship nomination and its subsequent description, one could test whether high barriers to cross-grade friendships might necessitate more intensive interaction for these relationships to exist at all. Similar in spirit, recent Bayesian models of informant accuracy incorporate whole-network dependencies (Butts 2003; Lee and Butts 2018; Marcum, Bevc, and Butts 2012), treating the "true" network as a collection of discrete latent ties. Extending the dyadic IRT approach to nominations would treat 
latent ties as inherently continuous phenomena—not on/off states—with nominations indicating a relationship meets a threshold for reporting.

Duncan (1985a, 1985b) emphasized that, in contrast to classical methods, IRT treats survey questions both as surrogates of respondent attitudes and as social objects worthy of study in their own right. In this sense, the dyadic IRT complements what Butts (2003) labels the "cognitivist" perspective on network reporting, contrasted with the "classical" perspective. Studies in the latter tradition presume a criterion structure (imposed by the analyst) that respondents are more or less accurate in reporting. ${ }^{19}$ The cognitivist perspective — associated with David Krackhardt (1987) — centers attention on perceived networks as social structures with their own force and effect. While the dyadic IRT approach does not necessitate a cognitivist perspective, it frees the analyst from the presumption of a criterion structure. Studies in the cognitivist tradition typically rely on multiple raters' descriptions of the same dyad via a single item, but they share with this paper-especially Section V-a concern with systematic variation in how reported ties relate to each other.

Some network instruments have a clear right or wrong answer. An Add Health respondent either visited their friend's home within the past week or they did not. However, criterion structures are more theoretically tenuous for affective relationships or seemingly objective interactions where the definition of the interaction is itself socially constructed (Bearman and Parigi 2004; Fischer 1982). For example, whether two

\footnotetext{
${ }^{19}$ Butts (2003) identifies his own work with the classical, non-cognitivist perspective.
} 
adolescents discussed a personal problem depends on their respective definitions of "personal problem." In IRT, while the latent trait represents a generative social process undergirding a response, models of item properties and item-by-respondent interactions (e.g. DIF) highlight the different ways elicited ties relate to each other and the shared underlying process. 


\section{$\underline{\text { References }}$}

Bearman, Peter and Paolo Parigi. 2004. "Cloning Headless Frogs and Other Important Matters: Conversation Topics and Network Structure.” Social Forces 83(2):535557.

Blei, David M., Alp Kucukelbir, and Jon D. McAuliffe. 2017. "Variational Inference: A Review for Statisticians." Journal of the American Statistical Association 112(518):859-77.

de Boeck, Paul and Mark Wilson. 2004. Explanatory Item Response Models: A Generalized Linear and Nonlinear Approach. Springer New York.

Buerkner, Paul-Christian. 2016. "Brms: An R Package for Bayesian Multilevel Models Using Stan.” Journal of Statistical Software 80(1):1-28.

Burt, Ronald S. 1997. “A Note on Social Capital and Network Content.” Social Networks 19(4):355-73.

Butts, Carter T. 2003. "Network Inference, Error, and Informant (in)Accuracy: A Bayesian Approach.” Social Networks 25(2):103-40.

Douglas, Jeffrey A., Louis A. Roussos, and William Stout. 1996. "Item-Bundle DIF Hypothesis Testing: Identifying Suspect Bundles and Assessing Their Differential Functioning." Journal of Educational Measurement 33(4):465-84. 
van Duijn, Marijtje, Tom AB Snijders, and Bonne JH Zijlstra. 2004. “P2: A Random Effects Model with Covariates for Directed Graphs.” Statistica Neerlandica 58(2):234-254.

Duncan, Otis Dudley. 1985a. "Measurement and Structure: Strategies for the Design and Analysis of Subjective Survey Data".” Pp. 179-229 in Surveying Subjective Phenomena. Vol. 1, edited by C. F. Turner and E. Martin. New York: Russell Sage Foundation.

Duncan, Otis Dudley. 1985b. "Rasch Measurement: Further Examples and Discussion." Pp. 367-403 in Surveying Subjective Phenomena. Vol. 2, edited by C. F. Turner and E. Martin. New York: Russell Sage Foundation.

Eagly, Alice H. and Valerie J. Steffen. 1984. "Gender Stereotypes Stem from the Distribution of Women and Men into Social Roles." Journal of Personality and Social Psychology 46(4):735.

Embretson, Susan E. 1996. “The New Rules of Measurement.” Psychological Assessment $8(4): 341-49$.

Ferligoj, Anuška and Valentina Hlebec. 1995. "Reliability of Network Measurements." Pp. 219-32 in Contributions to Methodology and Statistics: Proceedings of the International Conference on Statistics and Methodology, Bled, Slovenia, September 13 - 15, 1993, edited by A. Ferligoj and A. Kramberger. Ljubljana, Slovenia: University of Ljubljana Press. 
Ferligoj, Anuška and Valentina Hlebec. 1999. "Evaluation of Social Network Measurement Instruments.” Social Networks 21(2):111-30.

Fischer, Claude S. 1982. "What Do We Mean by 'Friend'? An Inductive Study.” Social Networks 3(4):287-306.

Fox, Jean-Paul. 2010. Bayesian Item Response Modeling: Theory and Applications. Springer.

Fox, Jean-Paul and Cees A. W. Glas. 2001. "Bayesian Estimation of a Multilevel IRT Model Using Gibbs Sampling.” Psychometrika 66(2):271-88.

Frank, Kenneth A., Chandra Muller, and Anna S. Mueller. 2013. "The Embeddedness of Adolescent Friendship Nominations: The Formation of Social Capital in Emergent Network Structures." American Journal of Sociology 119(1):216-53.

Granovetter, Mark S. 1973. "The Strength of Weak Ties.” American Journal of Sociology 78(6):1360-1380.

Hoff, Peter D. 2005. "Bilinear Mixed-Effects Models for Dyadic Data." Journal of the American Statistical Association 100(469):286-295.

Hoff, Peter D. 2009. "Multiplicative Latent Factor Models for Description and Prediction of Social Networks." Computational and Mathematical Organization Theory 15(4):261-72. 
Hoff, Peter, Bailey Fosdick, Alex Volfovsky, and Katherine Stovel. 2013. "Likelihoods for Fixed Rank Nomination Networks.” Network Science 1(3):253-77.

Hoffman, Matthew D. and Andrew Gelman. 2014. "The No-U-Turn Sampler: Adaptively Setting Path Lengths in Hamiltonian Monte Carlo." Journal of Machine Learning Research 15(1):1593-1623.

Iacobucci, Dawn, R. Neelamegham, and Nigel Hopkins. 1999. "Measurement Quality Issues in Dyadic Models of Relationships.” Social Networks 21(3):211-37.

Jeon, Minjeong and Paul de Boeck. 2016. "A Generalized Item Response Tree Model for Psychological Assessments." Behavior Research Methods 48(3):1070-85.

Koehly, Laura M. and Christopher Steven Marcum. 2018. “Multi-Relational Measurement for Latent Construct Networks.” Psychological Methods 23(1):4257.

Kogovšek, Tina, Anuška Ferligoj, Germa Coenders, and Willem E. Saris. 2002. "Estimating the Reliability and Validity of Personal Support Measures: Full Information ML Estimation with Planned Incomplete Data." Social Networks 24(1):1-20.

Krackhardt, David. 1987. “Cognitive Social Structures.” Social Networks 9(2):109-34. 
Kreager, Derek A. and Dana L. Haynie. 2011. "Dangerous Liaisons? Dating and Drinking Diffusion in Adolescent Peer Networks." American Sociological Review 76(5):737-63.

Lazega, Emmanuel and Marijtje van Duijn. 1997. "Position in Formal Structure, Personal Characteristics and Choices of Advisors in a Law Firm: A Logistic Regression Model for Dyadic Network Data.” Social Networks 19(4):375-397.

Lee, Francis and Carter T. Butts. 2018. "Mutual Assent or Unilateral Nomination? A Performance Comparison of Intersection and Union Rules for Integrating SelfReports of Social Relationships." Social Networks 55:55-62.

Lewandowski, Daniel, Dorota Kurowicka, and Harry Joe. 2009. “Generating Random Correlation Matrices Based on Vines and Extended Onion Method.” Journal of Multivariate Analysis 100(9):1989-2001.

MacCallum, Robert C., Michael W. Browne, and Hazuki M. Sugawara. 1996. "Power Analysis and Determination of Sample Size for Covariance Structure Modeling." Psychological Methods 1(2):130.

Marcum, Christopher Steven, Christine A. Bevc, and Carter T. Butts. 2012. "Mechanisms of Control in Emergent Interorganizational Networks." Policy Studies Journal 40(3):516-546.

Marsden, Peter V. and Karen E. Campbell. 1984. "Measuring Tie Strength.” Social Forces 63(2):482-501. 
McElreath, Richard. 2016. Rethinking: An R Package for Fitting and Manipulating Bayesian Models.

McFarland, Daniel A., James Moody, David Diehl, Jeffrey A. Smith, and Reuben J. Thomas. 2014. "Network Ecology and Adolescent Social Structure." American Sociological Review 79(6):1088-1121.

McNelles, Laurie R. and Jennifer A. Connolly. 1999. “Intimacy Between Adolescent Friends: Age and Gender Differences in Intimate Affect and Intimate Behaviors.” Journal of Research on Adolescence 9(2):143.

McPherson, Miller, Lynn Smith-Lovin, and Matthew E. Brashears. 2006. "Social Isolation in America: Changes in Core Discussion Networks over Two Decades." American Sociological Review 71(3):353-375.

Mead, George H. 1967. Mind, Self, and Society: From the Standpoint of a Social Behaviorist. Chicago: The University of Chicago Press.

Meulders, Michel and Yiyu Xie. 2004. "Person-by-Item Predictors.” Pp. 213-40 in Explanatory Item Response Models: A Generalized Linear and Nonlinear Approach, edited by P. de Boeck and M. Wilson. New York: Springer.

Newman, M. E. J. 2018. "Network Structure from Rich but Noisy Data.” Nature Physics 14(6):542. 
van den Noortgate, Wim and Paul de Boeck. 2005. “Assessing and Explaining Differential Item Functioning Using Logistic Mixed Models.” Journal of Educational and Behavioral Statistics 30(4):443-64.

Payne, Danielle C. and Benjamin Cornwell. 2007. "Reconsidering Peer Influences on Delinquency: Do Less Proximate Contacts Matter?” Journal of Quantitative Criminology 23(2):127-49.

Polson, Nicholas G. and James G. Scott. 2012. "On the Half-Cauchy Prior for a Global Scale Parameter.” Bayesian Analysis 7(4):887-902.

Raudenbush, Stephen W., Christopher Johnson, and Robert J. Sampson. 2003. “A Multivariate, Multilevel Rasch Model with Application to Self-Reported Criminal Behavior." Sociological Methodology 33(1):169-211.

Raudenbush, Stephen W. and Robert J. Sampson. 1999. "Ecometrics: Toward a Science of Assessing Ecological Settings, With Application to the Systematic Social Observation of Neighborhoods." Sociological Methodology 29(1):1-41.

Rose, Amanda J. and Karen D. Rudolph. 2006. "A Review of Sex Differences in Peer Relationship Processes: Potential Trade-Offs for the Emotional and Behavioral Development of Girls and Boys." Psychological Bulletin 132(1):98-131.

Rue, Håvard, Sara Martino, and Nicolas Chopin. 2009. “Approximate Bayesian Inference for Latent Gaussian Models by Using Integrated Nested Laplace 
Approximations." Journal of the Royal Statistical Society: Series B (Statistical Methodology) 71(2):319-92.

Rue, Håvard, Andrea Riebler, Sigrunn H. Sørbye, Janine B. Illian, Daniel P. Simpson, and Finn K. Lindgren. 2017. "Bayesian Computing with INLA: A Review." Annual Review of Statistics and Its Application 4(1):395-421.

Rusch, Thomas, Paul Benjamin Lowry, Patrick Mair, and Horst Treiblmaier. 2017. "Breaking Free from the Limitations of Classical Test Theory: Developing and Measuring Information Systems Scales Using Item Response Theory.” Information \& Management 54(2):189-203.

Small, Mario Luis. 2013. "Weak Ties and the Core Discussion Network: Why People Regularly Discuss Important Matters with Unimportant Alters." Social Networks 35(3):470-483.

Smith, Sanne, Daniel A. McFarland, Frank Van Tubergen, and Ineke Maas. 2016. "Ethnic Composition and Friendship Segregation: Differential Effects for Adolescent Natives and Immigrants." American Journal of Sociology 121(4):1223-72.

Snijders, Tom A. B. 2016. "The Multiple Flavours of Multilevel Issues for Networks." Pp. 15-46 in Multilevel Network Analysis for the Social Sciences. Cham, Switzerland: Springer. 
Snijders, Tom A. b. and David A. Kenny. 1999. “The Social Relations Model for Family Data: A Multilevel Approach.” Personal Relationships 6(4):471-86.

Stan Development Team. 2018. RStan: The R Interface to Stan. R Package Version 2.17. 3.

Verbrugge, Lois M. 1979. "Multiplexity in Adult Friendships.” Social Forces 57(4):1286-1309.

Wang, Xiaofeng, Yu Ryan Yue, and Julian J. Faraway. 2018. Bayesian Regression Modelling with INLA. Boca Raton, FL: CRC Press.

Warner, Rebecca M., David A. Kenny, and Michael Stoto. 1979. “A New Round Robin Analysis of Variance for Social Interaction Data." Journal of Personality and Social Psychology 37(10):1742-57.

Zijlstra, Bonne J. H., Marijtje A. J. van Duijn, and Tom A. B. Snijders. 2006. "The Multilevel P2 Model: A Random Effects Model for the Analysis of Multiple Social Networks." Methodology 42-47. 
Table 1. Illustrative Data Structure from a Single School: Number of Item Responses Across Directed Dyads

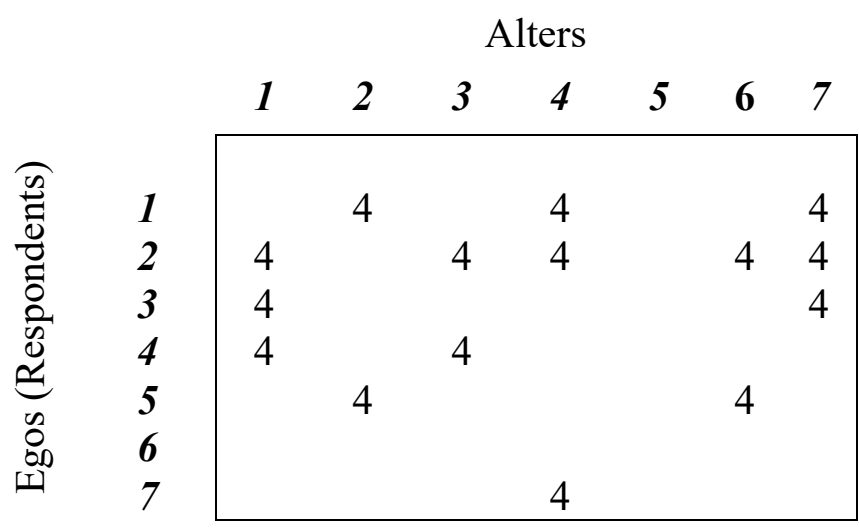


Table 2. Add Health Item Frequencies (Example School)

$$
\% \text { of Directed Nominations Listing Activity }
$$

Girl ego Girl Ego Boy ego Boy ego Girl alter Boy alter Girl alter Boy alter

Met alter after school to
hang out or go somewhere in last 7 days

$\begin{array}{lllll}67.5 & 28.2 & 40.9 & 50.7 & \mathbf{4 9 . 5}\end{array}$

Talked with alter on the telephone in last 7 days

$\begin{array}{lllll}56.7 & 32.3 & 37.0 & 45.7 & \mathbf{4 4 . 7}\end{array}$

Went to alter's house in last 7 days.

28.1

16.6

23.5

37.2

26.8

Spent time with alter last weekend.

27.6

28.5

37.7

32.9

Talked with alter about a problem in the last 7 days.

67.5

51.1

40.9

36.4

51.1

$\begin{array}{llllll}\text { N Dyads } & 2,135 & 1,371 & 1,302 & 1,496 & \mathbf{6 , 3 0 4}\end{array}$

$\mathrm{N}$ egos $=1,090 ; \mathrm{N}$ alters $=1,155$ 
Table 3. Result of Dyadic IRT Trait Decomposition Model in Example School

Posterior Mean

(Poster Std. Dev.)

$\begin{array}{lc}\text { Intercept } & 0.037 \\ \text { Relative Item Easiness }^{\text {a }} & (0.057) \\ \text { Talk on Phone } & \\ & \mathbf{- 0 . 3 1 9} \\ \text { Visit House } & \mathbf{( 0 . 0 4 6 )} \\ & \mathbf{- 1 . 6 1 8} \\ \text { Weekend } & \mathbf{( 0 . 0 5 1 )} \\ & \mathbf{- 1 . 1 4 5} \\ & \mathbf{( 0 . 0 4 9 )}\end{array}$

Posterior Means for Trait Random Components

[95-percent CI]

$\mathrm{SD}($ Ego)

1.162

$\mathrm{SD}($ Alter)

$[1.068,1.258]$

0.482

$\mathrm{SD}(\mathrm{Dyad})$

$[0.375,0.584]$

1.627

$[1.553,1.704]$

WAIC

25,475

N Egos

1,090

N Alters

1,155

N Dyads

6,304

N Item Responses

25,216

\footnotetext{
${ }^{a}$ Reference item: hang out or see each other after school in last seven days

Bold text for fixed effect: 95-percent credibility interval (CI) does not contain 0
} 
Table 4. Results of Dyadic IRT Models for Tie Strength

for 50 Schools without School Covariates

\begin{tabular}{|c|c|c|c|}
\hline & \multicolumn{3}{|c|}{$\begin{array}{c}\text { Poster Mean } \\
\text { (Posterior Std. Dev.) }\end{array}$} \\
\hline & $\begin{array}{c}\text { Trait Decomposition } \\
\text { Model }\end{array}$ & $\begin{array}{l}\text { Gender Mix } \\
\text { Model }\end{array}$ & $\begin{array}{c}\text { Gender Mix } \\
\text { with Actor/Dyad Controls }\end{array}$ \\
\hline Intercept & $\begin{array}{l}-0.561 \\
(0.065)\end{array}$ & $\begin{array}{c}-0.030 \\
(0.069)\end{array}$ & $\begin{array}{l}-\mathbf{- 0 . 2 3 3} \\
(\mathbf{0 . 0 6 3})\end{array}$ \\
\hline Ego girl/alter boy ${ }^{a}$ & & $\begin{array}{l}-1.372 \\
(0.018)\end{array}$ & $\begin{array}{l}-1.100 \\
(0.018)\end{array}$ \\
\hline Ego boy/alter girl ${ }^{\mathrm{a}}$ & & $\begin{array}{l}-1.121 \\
(0.024)\end{array}$ & $\begin{array}{c}-\mathbf{- 0 . 8 0 6} \\
(\mathbf{0 . 0 2 3})\end{array}$ \\
\hline Ego boy/alter boy ${ }^{a}$ & & $\begin{array}{c}-0.299 \\
(0.023)\end{array}$ & $\begin{array}{c}-0.136 \\
(0.022)\end{array}$ \\
\hline Same Grade & & & $\begin{array}{l}-0.789 \\
(0.016)\end{array}$ \\
\hline $\begin{array}{l}\text { Reciprocated } \\
\text { Nomination }\end{array}$ & & & $\begin{array}{c}1.145 \\
(0.013)\end{array}$ \\
\hline Ego $7^{\text {th }}$ or $8^{\text {th }}$ Grade & & & $\begin{array}{c}-0.326 \\
(0.075)\end{array}$ \\
\hline Ego $11^{\text {th }}$ or $12^{\text {th }}$ Grade & & & $\begin{array}{c}0.204 \\
(0.027)\end{array}$ \\
\hline Alter $7^{\text {th }}$ or $8^{\text {th }}$ Grade & & & $\begin{array}{l}-0.244 \\
(0.067)\end{array}$ \\
\hline Alter $11^{\text {th }}$ or $12^{\text {th }}$ Grade & & & $\begin{array}{c}0.001 \\
(0.021)\end{array}$ \\
\hline $\begin{array}{l}\text { Sqrt. Ego's In-degree } \\
\text { (Z-score) }\end{array}$ & & & $\begin{array}{c}0.145 \\
(0.011)\end{array}$ \\
\hline $\begin{array}{l}\text { Sqrt. Ego's Out-degree } \\
\text { (Z-score) }\end{array}$ & & & $\begin{array}{l}-0.171 \\
(0.012)\end{array}$ \\
\hline $\begin{array}{l}\text { Sqrt. Alter's In-degree } \\
\text { (Z-score) }\end{array}$ & & & $\begin{array}{l}-\mathbf{- 0 . 0 5 0} \\
(0.007)\end{array}$ \\
\hline $\begin{array}{l}\text { Sqrt. Shared Neighbors } \\
\text { (Z-score) }\end{array}$ & & & $\begin{array}{c}0.325 \\
(0.008)\end{array}$ \\
\hline
\end{tabular}


$\underline{\text { Table 4. Dyadic IRT Models for Tie Strength without School Covariates (cont.) }}$

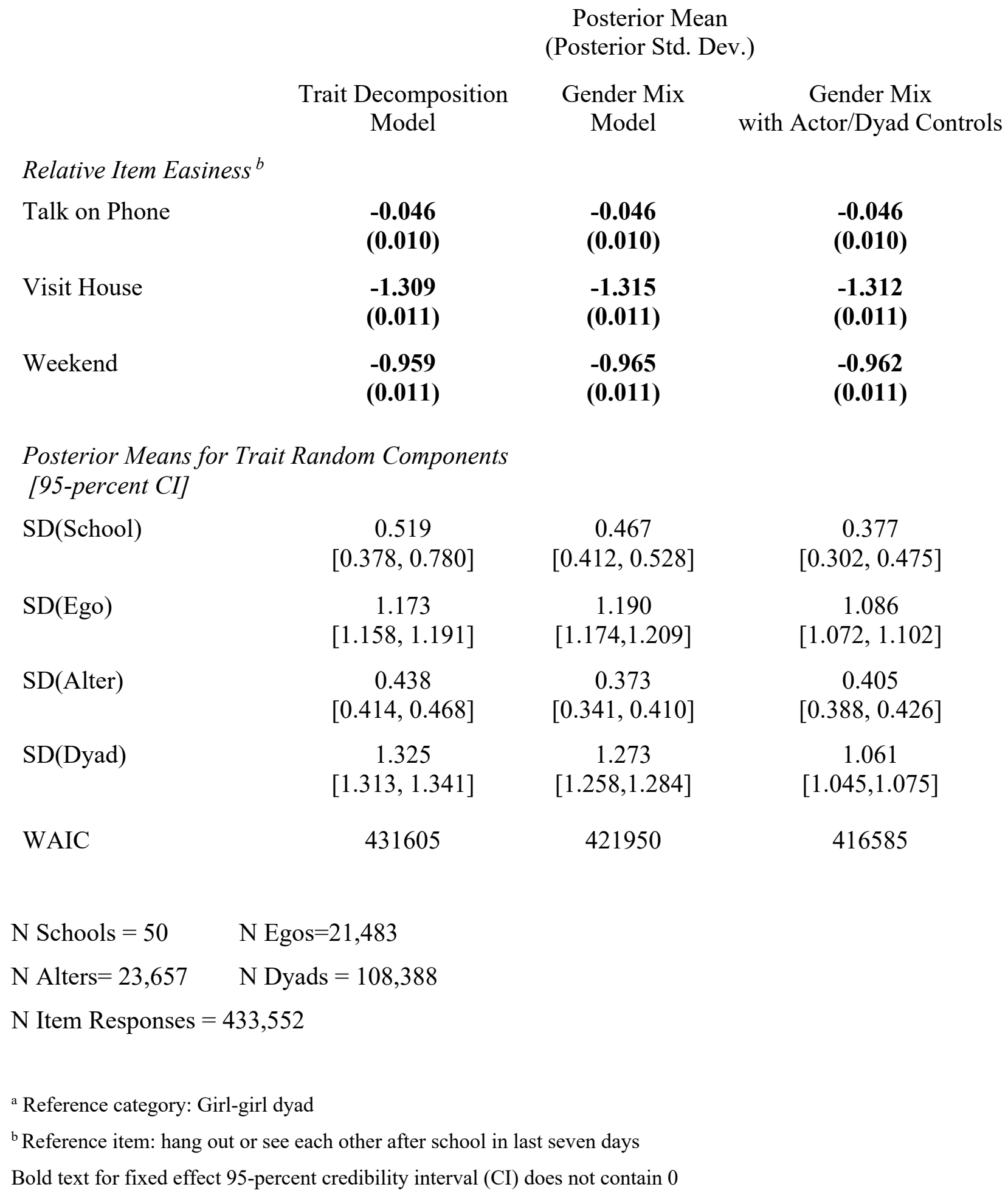

Posterior Mean

Posterior Std. Dev.

$-0.046$

$-1.312$

$\mathbf{- 0 . 9 5 9}$

$-0.965$

$-0.962$

(0.011)

Posterior Means for Trait Random Components [95-percent CI] 
Table 5. Results of Baseline Dyadic DIF Model

\begin{tabular}{|c|c|}
\hline & $\begin{array}{c}\text { Posterior Mean } \\
\text { (Posterior Std. Dev.) }\end{array}$ \\
\hline \multicolumn{2}{|l|}{ Parameters for Trait } \\
\hline Intercept & $\begin{array}{l}-0.080 \\
(0.070)\end{array}$ \\
\hline Ego girl/alter boy & $\begin{array}{l}-1.347 \\
(0.018)\end{array}$ \\
\hline Ego boy/alter girl & $\begin{array}{l}-1.147 \\
(0.025)\end{array}$ \\
\hline Ego boy/alter boy & $\begin{array}{l}-0.254 \\
(0.024)\end{array}$ \\
\hline \multicolumn{2}{|c|}{ "Discuss problems" Relative Easiness Parameters } \\
\hline Intercept & $\begin{array}{c}0.412 \\
(0.016)\end{array}$ \\
\hline Ego girl/alter boy & $\begin{array}{c}0.165 \\
(0.024)\end{array}$ \\
\hline Ego boy/alter girl & $\begin{array}{l}-0.552 \\
(0.025)\end{array}$ \\
\hline Ego boy/alter boy & $\begin{array}{l}-1.550 \\
(0.022)\end{array}$ \\
\hline \multicolumn{2}{|c|}{ Relative Easiness for Other Items } \\
\hline Talk on Phone & $\begin{array}{l}-0.047 \\
(0.011)\end{array}$ \\
\hline Visit House & $\begin{array}{l}-1.328 \\
(0.011)\end{array}$ \\
\hline Weekend & $\begin{array}{c}-0.975 \\
(0.011)\end{array}$ \\
\hline
\end{tabular}


Table 5. Results of Baseline Dyadic DIF Model (cont.)

Posterior Means of Trait Random Components [95-percent CI]

$\begin{array}{lc}\mathrm{SD}(\text { School) } & 0.448 \\ & {[0.369,0.529]} \\ \mathrm{SD}(\text { Ego }) & 1.260 \\ \mathrm{SD}(\text { Alter }) & {[1.242,1.276]} \\ & 0.311 \\ \mathrm{SD} \text { (Dyad) } & {[0.283,0.345]} \\ & 1.331 \\ & {[1.317,1.342]}\end{array}$

$W A I C$

522082

\footnotetext{
$\mathrm{N}$ Schools $=50 \quad \mathrm{~N}$ Egos $=21,483$

N Alters $=23,657 \quad$ N Dyads $=108,388$;

$\mathrm{N}$ Item Responses $=541,940$

${ }^{\text {a }}$ Reference category: Girl-girl dyad

${ }^{b}$ Reference item: hang out or see each other after school in last seven days

Bold text for fixed effect 95-percent credibility interval (CI) does not contain 0
} 


\section{Table 6. UCDS Personality Items}

\begin{tabular}{llc} 
Latent Trait & Alter is... & \% Directed Dyads Attributing \\
\hline \multirow{2}{*}{ Instrumentality } & & \\
& & \\
& Strong & 14.46 \\
& Decisive & 14.04 \\
& Dominant & 10.28 \\
& $\begin{array}{l}\text { Influential/translates ideas into } \\
\text { action }\end{array}$ & 13.64 \\
& & \\
& & \\
& Lovpressiveness & \\
& Supportive & 15.47 \\
& $\begin{array}{l}\text { Intuitive/good at helping resolve } \\
\text { emotional problems }\end{array}$
\end{tabular}

$\mathrm{N}$ dyads $=3,277 ; \mathrm{N}$ attributees $=412 ; \mathrm{N}$ attributors $=350 ; \mathrm{N}$ groups $=44$ 
Table 7. Results of Multidimensional Dyadic Trait

Decomposition Model of Instrumentality and Expressiveness

Trait Intercept

Posterior Means

(Posterior SD)

Parameters

Instrumentality

$-2.762$

(0.128)

Expressiveness

$-2.392$

(0.139)

\section{Relative Item}

Easiness Parameters

Instrumentality Items

$\begin{array}{cc}\text { Strong } & 0.100 \\ & (0.085) \\ \text { Decisive } & 0.049 \\ & (0.086) \\ \text { Dominant } & \mathbf{- 0 . 4 5 2} \\ & \mathbf{( 0 . 0 9 3 )}\end{array}$

Expressiveness Items

$\begin{array}{cc} & \mathbf{0 . 2 8 7} \\ \text { Supportive } & \mathbf{( 0 . 0 8 1 )} \\ & \\ \text { Intuitive } & \mathbf{- 0 . 4 3 3} \\ & \mathbf{( 0 . 0 8 8 )}\end{array}$

Posterior Means of Trait Random Components [95-percent CI]

Dyad

$\begin{array}{rc}\mathrm{SD} \text { (Instrumentality) } & 0.783 \\ & {[0.633,0.927]} \\ \mathrm{SD} \text { (Expressiveness) } & 1.053 \\ & {[0.889,1.213]} \\ \text { Corr(Instr, Expr) } & .414 \\ & {[.207, .622]}\end{array}$


Table 7. Results of Multidimensional Trait

Decomposition Models (cont.)

\section{Posterior Means of Trait Random Components [95-percent CI]}

$\begin{array}{rc}\text { Attributee (Alter) } & \\ \text { SD(Instrumentality) } & 1.676 \\ & {[1.501 .1 .864]} \\ \text { SD(Expressiveness) } & 1.260 \\ \text { Corr(Instr, Expr) } & {[1.106,1.432]} \\ & .468 \\ \text { Attributor (Ego) } & {[.343, .582]} \\ \text { SD(Instrumentality) } & 0.810 \\ \text { SD(Expressiveness) } & {[0.692,0.938]} \\ & 0.929 \\ \text { Corr(Instr, Expr) } & {[0.786,1.082]} \\ & .840 \\ \text { Group } \quad[.712, .946] \\ \text { SD(Expressiveness) } & \\ & 0.459,0.713] \\ \text { WAIC } & 14261\end{array}$

$\mathrm{N}$ groups $=44 \quad \mathrm{~N}$ dyads $=3,277$

$\mathrm{N}$ attributees $=412 \quad \mathrm{~N}$ attributors $=350$

$\mathrm{N}$ Item Responses $=22,939$

Bold text for fixed effects: 95-percent credibility interval (CI) does not contain 0.

Italicized text indicates 90-percent CI does not contain zero but 95-percent CI does not contain 0. 
Figure 1. Item Characteristic Curves from Two-parameter Logistic Model

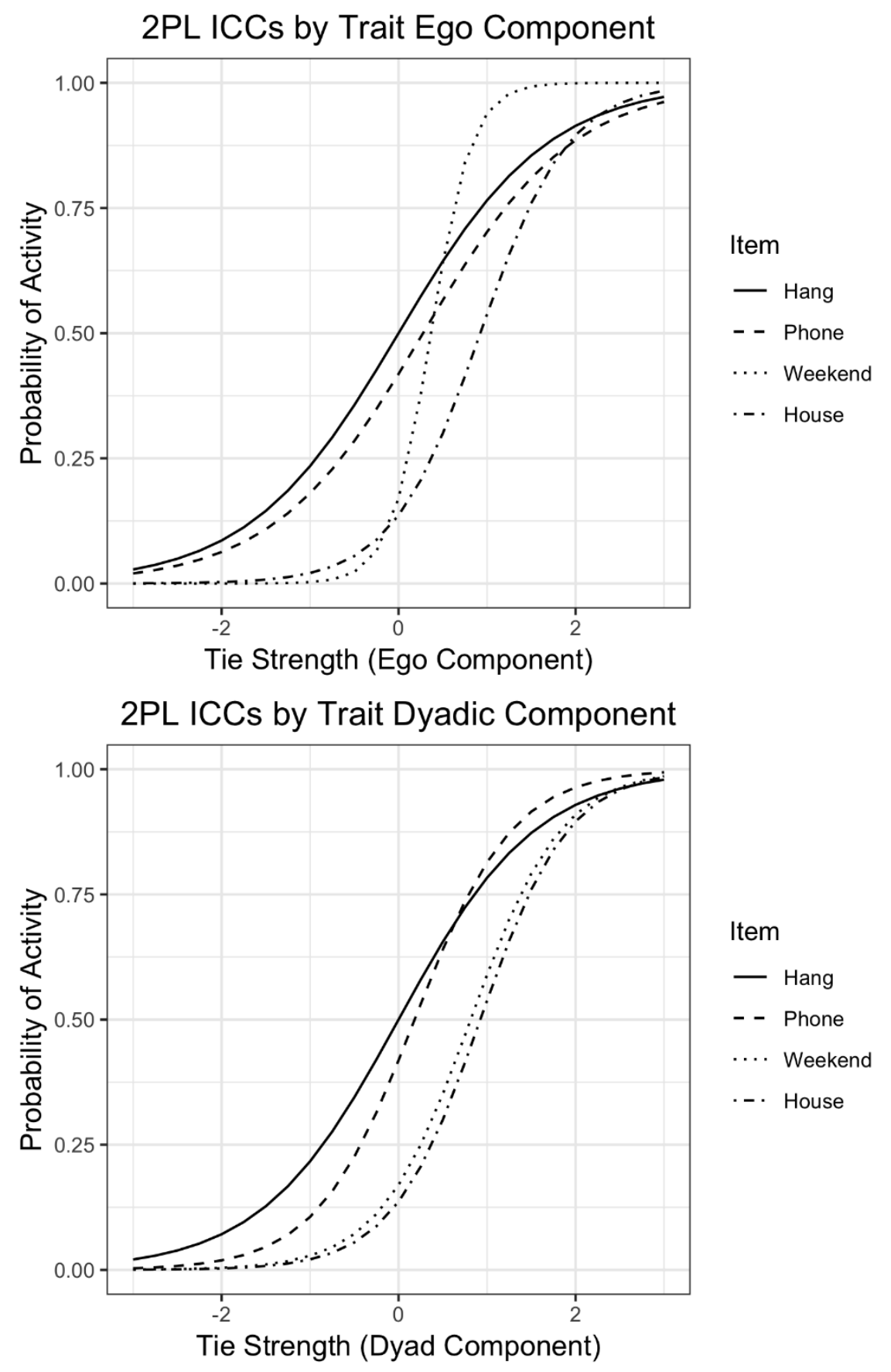


Figure 2. Fitted Latent Tie Strength by Dyad Gender Mix

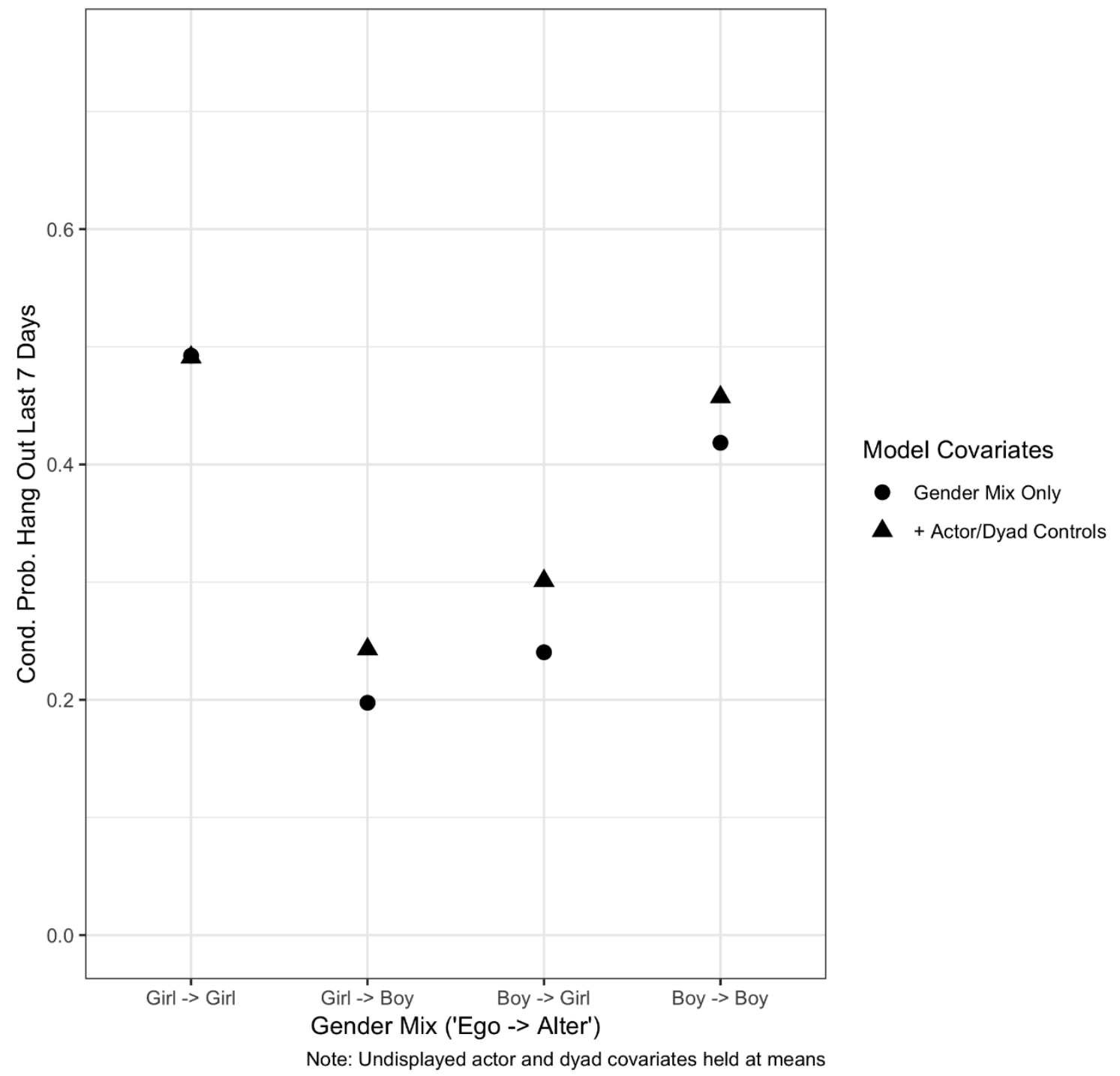


Figure 3. Fitted Latent Tie Strength by Gender Mix and School Size

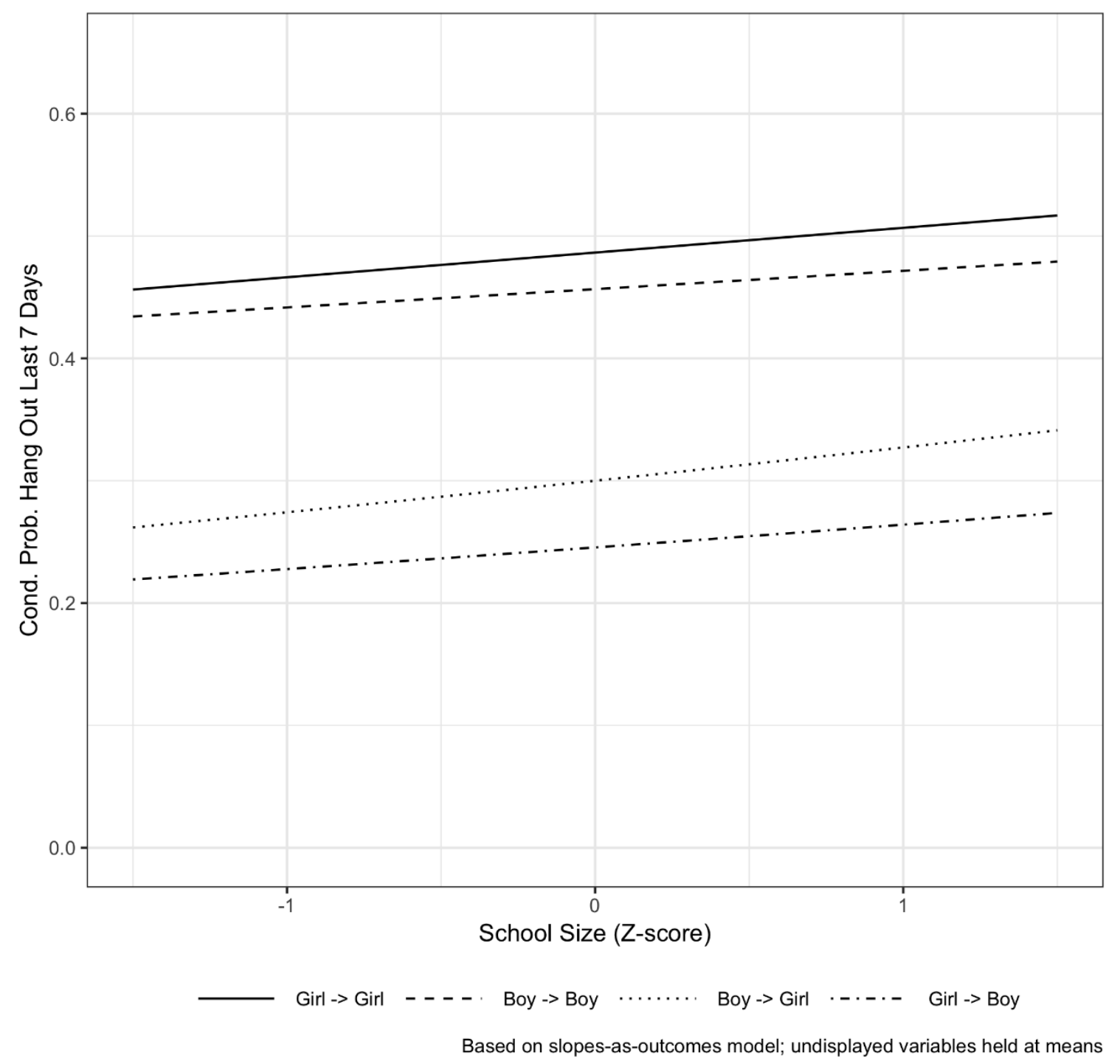


Figure 4. Discussing Personal Problems Fitted DIF by School Gender Heterogeneity

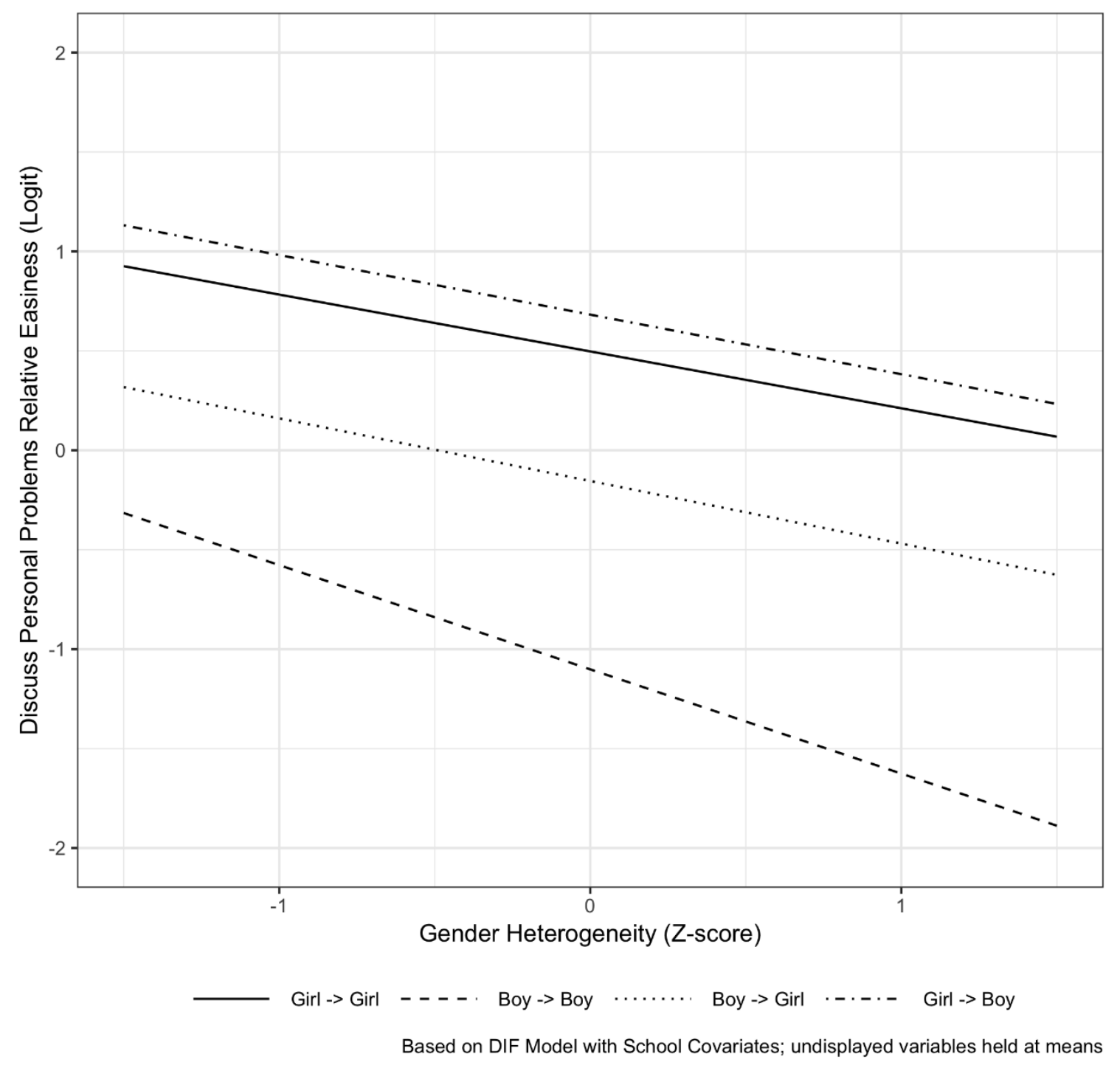


Figure 5. Fitted Latent Tie Strength: High School vs. Middle School

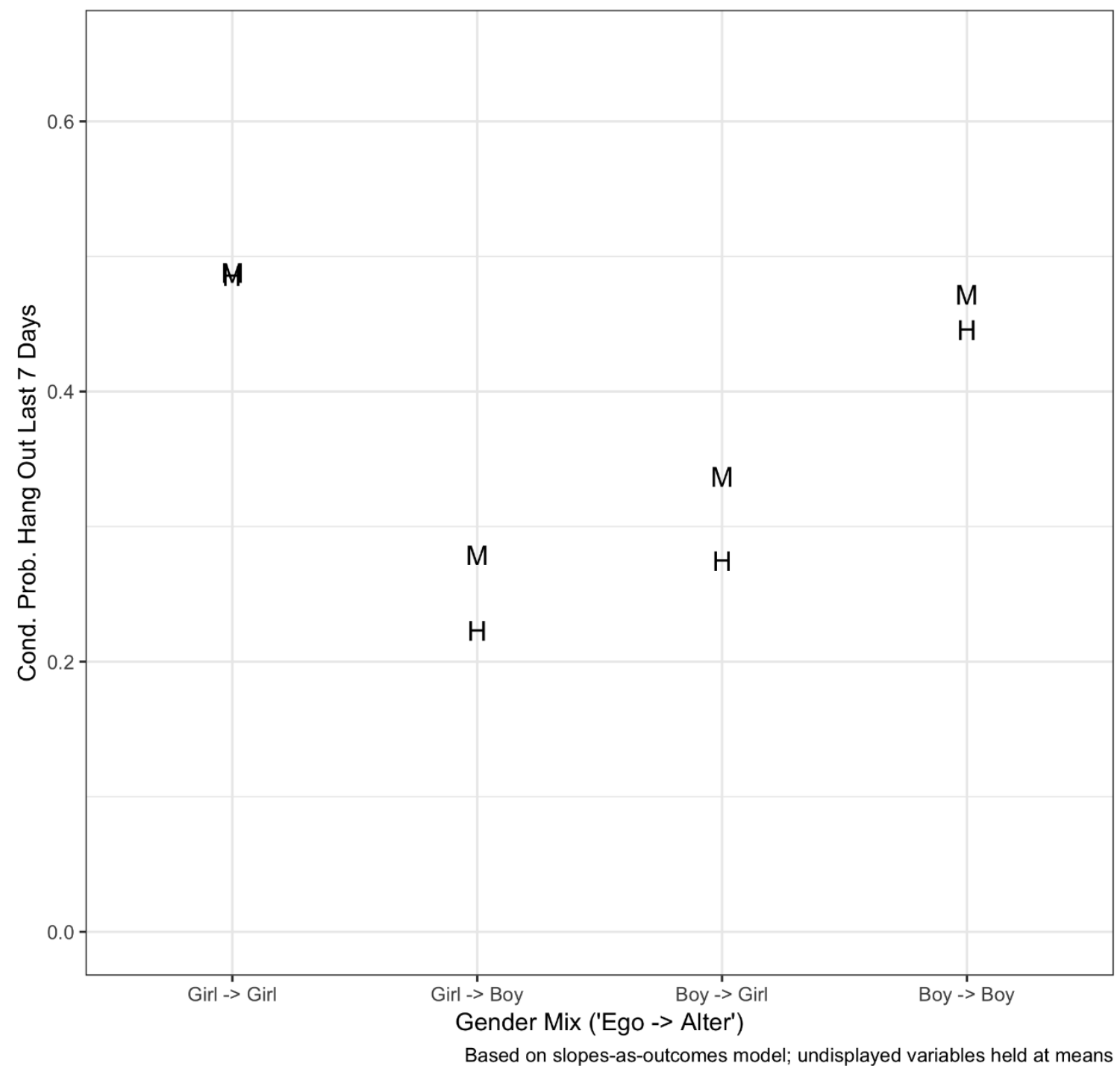


Figure 6. Example Item Characteristic Curves Illustrating Differential Item Functioning

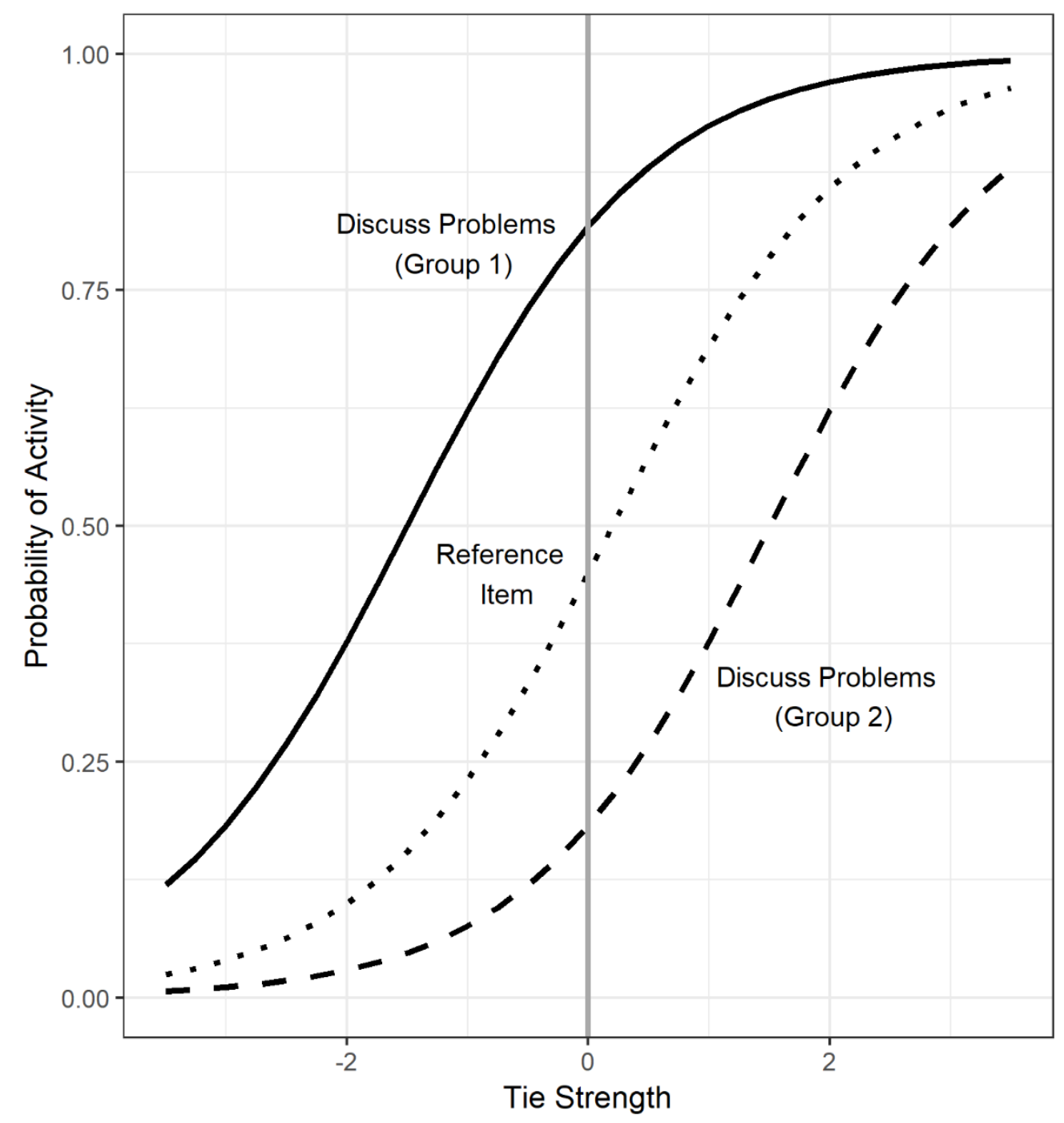


Figure 7. Discussing Personal Problems Fitted DIF by Dyad Gender Mix

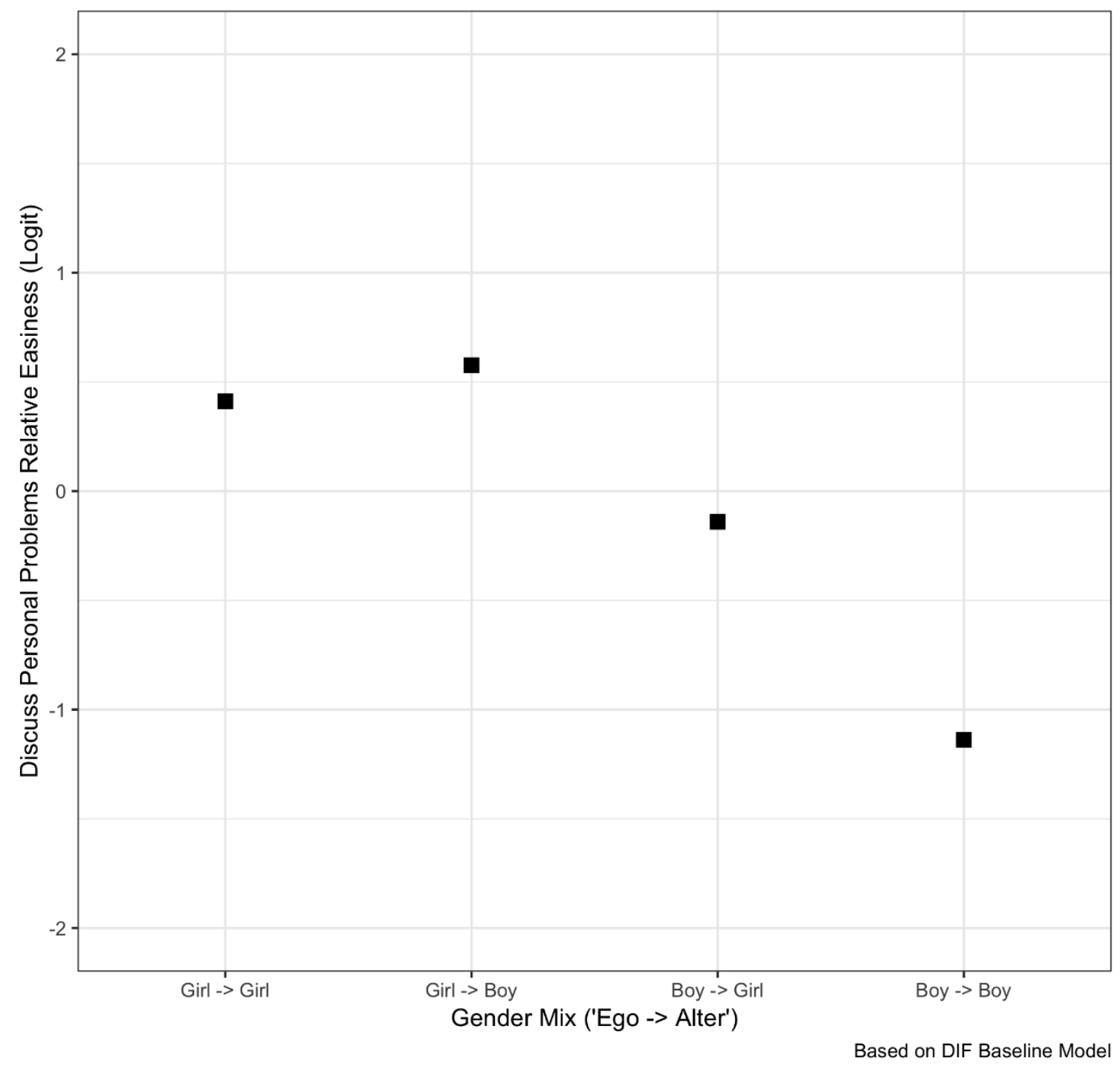


Figure 8. Discussing Personal Problems Fitted DIF by School Size

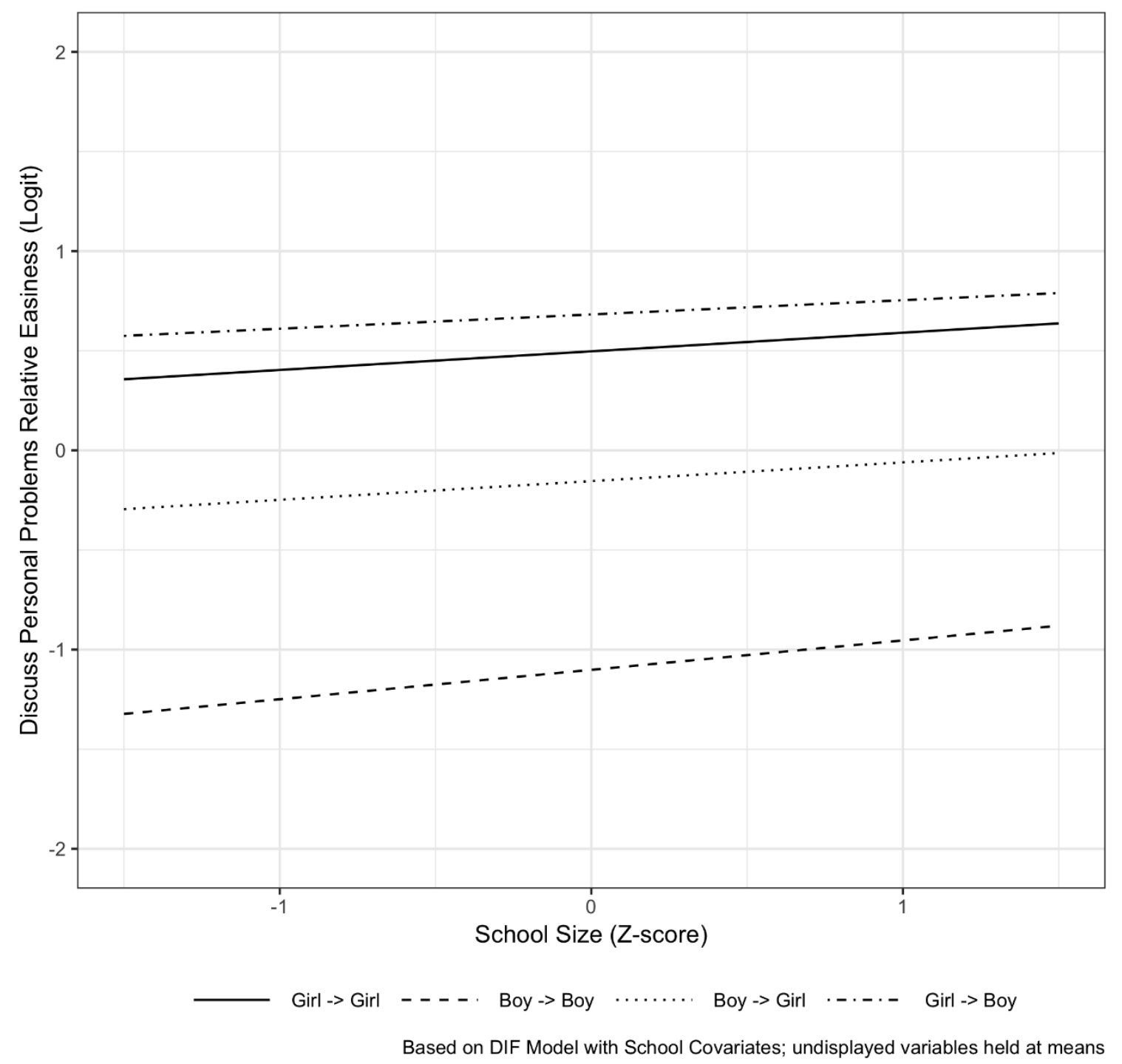


Figure 9. Discussing Personal Problems Fitted DIF by Gender Heterogeneity

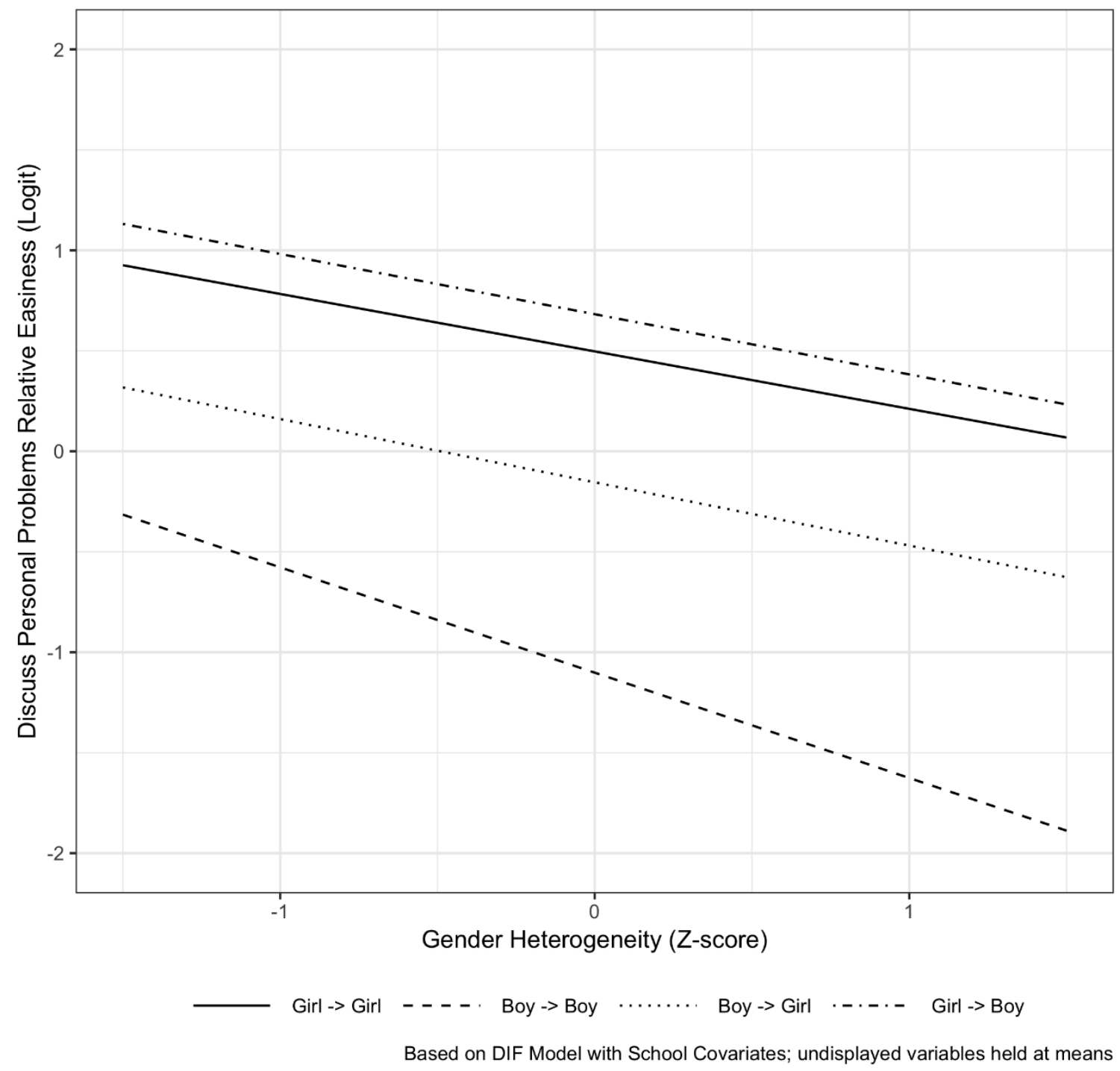


Figure 10. Discussing Personal Problems Fitted DIF: High School vs. Middle School

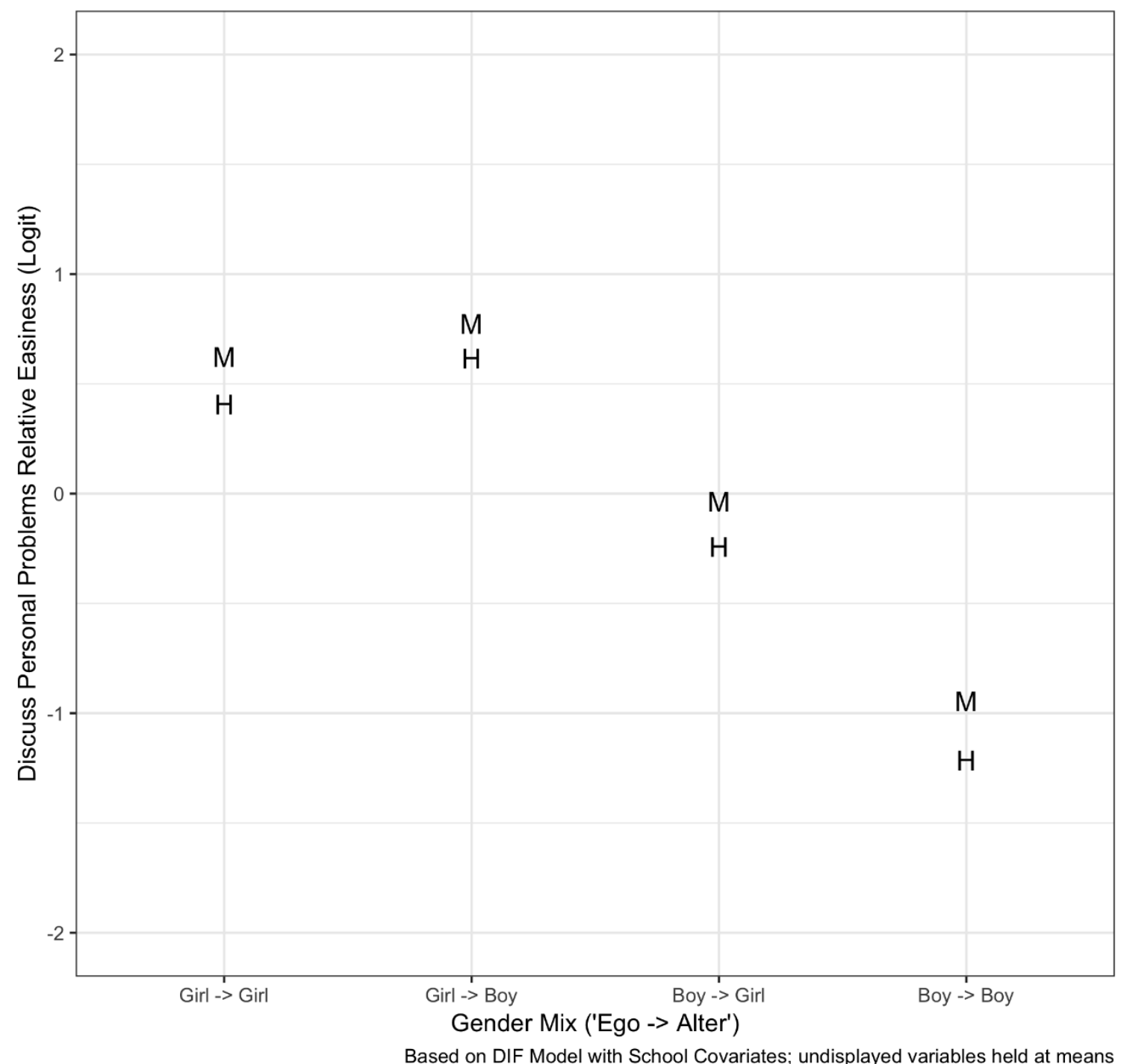

